\title{
Composite fermion nonlinear sigma models
}

\author{
Chao-Jung Lee $\odot$ \\ Department of Physics, California Institute of Technology, Pasadena, California 91125, USA \\ Prashant Kumar \\ Department of Physics, Princeton University, Princeton, New Jersey 08544, USA \\ Michael Mulligan \\ Department of Physics and Astronomy, University of California, Riverside, California 92511, USA
}

(Received 16 February 2021; accepted 27 August 2021; published 13 September 2021)

\begin{abstract}
We study particle-hole symmetry at the integer quantum Hall plateau transition using composite fermion mean-field theory. Because this theory implicitly includes some electron-electron interactions, it also has applications to certain fractional quantum Hall plateau transitions. Previous work [P. Kumar et al., Phys. Rev. B 100, 235124 (2019)] using this approach showed that the diffusive quantum criticality of this transition is described by a nonlinear sigma model with topological $\theta=\pi$ term. This result, which holds for both the Dirac and Halperin, Lee, and Read composite fermion theories, signifies an emergent particle-hole (reflection) symmetry of the integer (fractional) quantum Hall transition. Here we consider the stability of this result to various particle-hole symmetry-violating perturbations. In the presence of quenched disorder that preserves particle-hole symmetry, we find that finite longitudinal conductivity at this transition requires the vanishing of a symmetry-violating composite fermion effective mass, which if present would generally lead to $\theta \neq \pi$ and a corresponding violation of particle-hole symmetric electrical transport $\sigma_{x y} \neq \frac{1}{2} \frac{e^{2}}{h}$. When the disorder does not preserve particle-hole symmetry, we find that $\theta$ can vary continuously within the diffusive regime. Our results call for further study of the universality of the quantum Hall plateau transition.
\end{abstract}

DOI: 10.1103/PhysRevB.104.125119

\section{INTRODUCTION}

\section{A. Overview}

Electrons in two spatial dimensions exhibit quantized Hall plateaus when their density is comparable to the strength of a transverse magnetic field [1,2]. Within the picture of disordered noninteracting electrons-appropriate for the integer quantum Hall effect [3] - these plateaus are due to the localization of all electronic states, except for those lying at a single energy level within each Landau level [4]. An integer quantum Hall plateau transition occurs as the electron chemical potential is tuned through this critical energy, typically by varying the magnetic field at fixed electron density [5]. While accounting for the basic phase structure, this description has some unsatisfactory features, in relation to the observed quantum critical phenomena [6]. Because the set of delocalized states is of measure zero, interactions must be included for nonzero finite-temperature longitudinal conductivity [7]. The numerically calculated localization length critical exponent $v_{\text {calc }}$ (varying from about 2.48-2.62 with the specific lattice model and calculation details [8-16]) do not appear to lie within the error bars of the measured exponent $v \approx 2.38[17,18]$. (Some recent works are critical of these theoretical results: inclusion of additional types of disorder may be relevant [19] and/or the critical scaling regime may require significantly larger systems sizes [20]; see also [21,22].) Furthermore, the difference of the calculated and measured dynamical critical exponents $z_{\text {calc }}-z \approx 1$ [23]. These disagreements are not entirely surprising since the Coulomb interaction is expected to be a relevant perturbation of the noninteracting fixed point $[24,25]$. Thus, a faithful model of the integer quantum Hall transition is thought to be interacting.

Composite fermions [26,27] provide a powerful dual framework for the quantum Hall effect. Intuitively, this duality proceeds by an exact transformation of the electron wave function by "flux attachment" [28]. A $(2+1)$ d effective field theory implementing this transformation consists of a composite fermion coupled to a Chern-Simons gauge field whose level is determined by the electron filling fraction [29-31]. A large variety of integer and fractional quantum Hall effects can then be understood within a composite fermion mean-field theory-in which fluctuations of this Chern-Simons gauge field and all other interactions are ignored-as integer quantum Hall states of composite fermions. Although inadequate to fully account for the effects of electron-electron interactions, e.g., $v_{\text {calc }} \approx 2.56$ and $z_{\text {calc }}=2$ [32], semiclassical reasoning and numerical calculations suggest that composite fermion mean-field theory provides a unified framework for the quantum Hall plateau transitions [33-35], hosting delocalized states for a range of critical energies [36,37].

This paper is concerned with an analytical description of the plateau transition using composite fermions and, in 
particular, any symmetry it might exhibit. We focus on the integer quantum Hall transition, however, our results can be readily adapted to certain fractional quantum Hall plateau transitions [38]. The relevant symmetry is therefore particlehole symmetry [39]; the generalization of this symmetry to appropriate fractional quantum Hall transitions is known as reflection symmetry $[38,40-42]$. In the limit of infinite cyclotron frequency, the particle-hole symmetry transformation relates states at electron filling fractions $v \leftrightarrow 1-v$ (in lowest Landau level). This symmetry is not present in the microscopic electron Hamiltonian. Nevertheless, evidence for an emergent particle-hole symmetry is seen in electrical transport measurements that find the DC Hall conductivity at $v=1 / 2$ to equal $\sigma_{x y}=\frac{1}{2} \frac{e^{2}}{h}$ (see $[43,44]$ and references therein). In the composite fermion theory of Halperin, Lee, and Read (HLR) [31], this electrical Hall conductivity requires [45] (and occurs $[37,46-48]$ in the presence of weak disorder $\rho_{x x} \neq 0$ ) a composite fermion Hall conductivity $\sigma_{x y}^{\text {cf }}=-\frac{1}{2} \frac{e^{2}}{h}$ [49]. From the point of view of composite fermion mean-field theory, particle-hole symmetry helps to explain the diffusive quantum criticality of the plateau transition: Because localized free fermions necessarily exhibit integral Hall conductivity [3], the half-integer value of $\sigma_{x y}^{\mathrm{cf}}$ (in units of $e^{2} / h$ ) implies the critical states are delocalized [50]. Our interest in this paper is to better understand whether particle-hole symmetric $\sigma_{x y}^{\text {cf }}$ is necessary for the diffusive quantum criticality. In particular, how universal is particle-hole symmetry to the integer quantum Hall plateau transition?

\section{B. Detailed summary}

We now provide a more detailed overview of this paper. There are two types of composite fermions theories for electrons at $v=1 / 2$ : the HLR theory [31], which involves a nonrelativistic composite fermion coupled to a Chern-Simons gauge field; and the Dirac composite fermion theory, introduced by Son [46] and further developed in a number of subsequent works [47,51-53], in which the nonrelativistic composite fermion is replaced by a Dirac fermion. Using a standard Dirac notation, the mean-field Lagrangians for HLR $(\eta=1)$ and Dirac $(\eta=0)$ composite fermion theories can be expressed as (see Sec. II)

$$
\mathcal{L}=\bar{\Psi}(i \not \partial+\not l) \Psi-\Psi^{\dagger} P_{2}\left(i \eta \partial_{0}+a_{0}\right) \Psi+m_{1} \Psi^{\dagger} \Psi+m_{2} \bar{\Psi} \Psi .
$$

Here $\Psi$ is a two-component Dirac fermion; $a_{0}(\mathbf{x})$ and $a_{j}(\mathbf{x})$ for $j \in\{1,2\}$ are possible uncorrelated quenched scalar and vector potential disorders (probing electromagnetic fields are set to zero); $P_{2}$ projects onto the second component of $\Psi ; m_{1}$ is a chemical potential that fixes the nonzero composite fermion density; and $m_{2}$ is a possible mass term. In this formulation (1.1), particle-hole symmetry acts as time-reversal invariance. Consequently, the terms involving $P_{2}$ and $m_{2}$ violate particlehole symmetry. The vector potential disorder $a_{j}(\mathbf{x})$ preserves particle-hole symmetry for an appropriate choice of disorder ensemble (see Sec. II A).

The numerical works $[36,37]$ that found the HLR composite fermion mean-field theory to exhibit an integer quantum Hall plateau transition with particle-hole symmetric transport at $v=1 / 2$, assumed a particle-hole symmetric ensemble for $a_{j}(\mathbf{x})$ and set $a_{0}(\mathbf{x})$ to zero. Since $m_{2}$ is generally nonzero in the HLR theory, this result is consistent with an emergent particle-hole symmetry. The Dirac composite fermion mean-field theory (1.1) manifestly has particle-hole symmetry so long as time-reversal invariance is preserved. Significant progress towards an analytical description of this transition was then made in [54], where it was shown that, for particle-hole symmetric disorder and when $m_{2}=0$, the DC zero-temperature electrical conductivity of composite fermions is encoded in the nonlinear sigma model (NLSM),

$$
S_{\mathrm{NLSM}}=\int d^{2} x \operatorname{Tr}\left(\frac{1}{2 g}\left(\partial_{j} Q\right)^{2}+i \frac{\theta}{16 \pi} \epsilon_{i j} Q \partial_{i} Q \partial_{j} Q\right),
$$

with $\theta=\pi$. Here the matrix boson $Q \in U(2 n) / U(n) \times U(n)$ parametrizes composite fermion charge density fluctuations with the replica limit $n \rightarrow 0$ to be taken at the end of any calculation using $S_{\mathrm{NLSM}}$. The marginally relevant coupling [55] (in the renormalization group sense), $g \propto 1 / \sigma_{x x}^{\text {cf }}$, characterizes the evolution from the ballistic to the diffusive regime of finite (impurity-averaged) conductivity $\sigma_{i j}^{\text {cf }}$ (henceforth measured in units of $e^{2} / h$ ). If only the first term in $S_{\mathrm{NLSM}}$ is present, the marginal relevancy of $g$ implies "weak" localization $\sigma_{x x}^{\text {cf }} \rightarrow 0$ in the thermodynamic limit. The topological $\theta$ term [56] is believed to prevent this localization when $\theta=\pi$ and (if unrenormalized) thereby explain the quantum critical diffusion [57-63]. As such, $\theta$ and $\sigma_{x y}^{\text {cf }}$ are related [54,57,61]:

$$
\theta=\pi+2 \pi\left(\sigma_{x y}^{\mathrm{cf}}-\frac{\eta}{2}\right) \bmod 2 \pi .
$$

Here $\sigma_{x y}^{\text {cf }}$ denotes either the HLR or Dirac DC composite fermion Hall conductivity, with particle-hole symmetric values $\sigma_{x y}^{\text {cf }}=-\frac{\eta}{2}$. Two features of this NLSM are worth emphasizing: first, it is identical in form to that argued by Pruisken et al. from the (dual) electron perspective [57,58]; second, $\theta=\pi$ regardless of whether or not $\eta$ is nonzero. [Intuitively, this occurs because the dependence on $\eta$ drops out in the DC limit in which the time derivative in (1.1) can be ignored.] Thus, in the presence of particle-hole symmetric disorder, the HLR and Dirac composite fermion mean-field theories are controlled by the same IR fixed point.

In this paper we generalize [54] to include uniform $m_{2}$ and random $a_{0}(\mathbf{x})$. First for vanishing $a_{0}(\mathbf{x})$ and when particlehole symmetric disorder $a_{i}(\mathbf{x})$ is present, we find that finite $\sigma_{x x}^{\text {cf }}$ requires the renormalized $m_{2}=0$. The resulting NSLM reduces to that found in [54] in which a $\theta=\pi$ term appears. We interpret this as an emergent particle-hole symmetry and the irrelevance of $m_{2}$ near the diffusive quantum critical point. When $a_{0}(\mathbf{x})$ is included, we find that the topological $\theta$ term varies continuously with the strength of this particle-hole symmetry-violating disorder, consistent with the simultaneous generation of nonzero symmetry-violating $m_{2}$. Because the NLSM formulation is controlled only for $\sigma_{x x}^{\text {cf }} \gg 1$ and the beta functions that determine the two-parameter flow of $1 / g$ and $\theta$ are nonperturbative [64], we are unable here to determine whether the ultimate low-energy fixed point of the sigma model with $\theta \neq \pi$ is an insulator or a quantum critical metal with varying Hall conductivity.

The remainder of this paper is organized as follows. In Sec. II we review the HLR and Dirac composite fermion 
mean-field theories with the aim of deriving the mean-field Lagrangian (1.1). In Sec. III A we construct the generating functional for disorder-averaged products of retarded and advanced composite fermion Green's functions. Encoded in this generating functional are observables such as the composite fermion density of states and conductivity. In Sec. III B we derive the NLSM for composite fermion mean-field theory, focusing on the topological $\theta$ term. This NLSM contains the effects of particle-hole preserving and particle-hole breaking disorders. In Sec. IV we conclude with a discussion of our results and of possibilities for future work. Appendices supplement arguments in the main text. Unless stated otherwise we take $e^{2}=\hbar=1$.

\section{COMPOSITE FERMION MEAN-FIELD THEORY}

We begin with a derivation of the mean-field Lagrangian (1.1) for the HLR and Dirac composite fermion theories.

\section{A. HLR mean-field theory}

The HLR Lagrangian is [31]

$$
\begin{aligned}
\mathcal{L}_{\mathrm{HLR}}= & \psi^{\dagger}\left(i \partial_{0}+A_{0}+V+a_{0}-\frac{\left(i \partial_{j}+A_{j}+a_{j}\right)^{2}}{2 m}\right) \psi \\
& +\frac{\epsilon^{\mu \nu \rho}}{8 \pi} a_{\mu} \partial_{\nu} a_{\rho}+\cdots .
\end{aligned}
$$

Here $\psi^{\dagger}(t, \mathbf{x})$ creates an HLR composite fermion; $A_{\mu}(t, \mathbf{x})$ is the external electromagnetic field; $a_{\mu}(t, \mathbf{x})$ is a dynamical $(2+1) \mathrm{d}$ Chern-Simons gauge field; $m$ is an effective mass, $\epsilon^{\mu \nu \rho}$ with $\mu, v, \rho \in\{0,1,2\}$ is the antisymmetric symbol with $\epsilon^{012}=1$; and the "..." include all other possible couplings and interactions, which we set to zero in the remainder. A nonzero uniform magnetic field $\epsilon_{i j} \partial_{i} A_{j}=B>0$ is assumed such that the electron filling fraction $v=1 / 2$. Variation of $\mathcal{L}_{\mathrm{HLR}}$ with respect to $A_{0}$ implies the electron and composite fermion densities are equal. $V(\mathbf{x})$ represents possible quenched electron scalar potential disorder. In this paper we assume that $V(\mathbf{x})$ is particle-hole symmetric, i.e., the disorder ensemble for $V(\mathbf{x})$ has vanishing odd moments $\overline{V\left(\mathbf{x}_{1}\right) \cdots V\left(\mathbf{x}_{2 p+1}\right)}=0$.

The mean-field approximation can be divided into two steps. The first is to impose the $a_{0}$ equation of motion, i.e., flux attachment,

$$
\psi^{\dagger} \psi=-\frac{1}{4 \pi} \epsilon^{i j} \partial_{i} a_{j} .
$$

This results in an effective magnetic field $B+\epsilon_{i j} \partial_{i} a_{j}$ that vanishes on average at half-filling, which we accommodate by shifting $A_{i}+a_{i} \rightarrow a_{i}$. Flux attachment (2.2) also implies that weak (electron) scalar potential disorder $V(\mathbf{x})$ is realized as anticorrelated vector and scalar potential randomness $[48,65]$ :

$$
\epsilon_{i j} \partial_{i} a_{j}(\mathbf{x})=-2 m V(\mathbf{x}) .
$$

The second (and final) step of the mean-field approximation is to set all dynamical fluctuations of $a_{\mu}(t, \mathbf{x})$ to zero. Thus, $a_{\mu}(\mathbf{x})$ is a background field in the mean-field approximation.

We choose a disorder ensemble for $V(\mathbf{x})$ such that the resulting vector potential $a_{j}(\mathbf{x})$ determined by (2.3) is a transverse Gaussian random variable with zero mean and variance
$W>0$

$$
\overline{a_{i}(\mathbf{x})}=0, \quad \overline{a_{i}(\mathbf{x}) a_{j}\left(\mathbf{x}^{\prime}\right)}=W \delta_{i j} \delta\left(\mathbf{x}-\mathbf{x}^{\prime}\right) .
$$

Such delta-function correlated vector potentials (2.4) arise from power-law correlated scalar potentials $\overline{V(\mathbf{x}) V\left(\mathbf{x}^{\prime}\right)} \propto$ $\left|\mathbf{x}-\mathbf{x}^{\prime}\right|^{-4}$ [66]. We remark that nonrelativistic fermions coupled to power-law correlated vector potential disorder without any scalar potential disorder obtain singular single-particle properties; two-particle properties remain regular and appear to coincide with delta-function correlated vector potential disorder [67-69].

Quenched disorder satisfying (2.3) appears to be necessary for particle-hole symmetric composite fermion Hall conductivity $\sigma_{x y}^{\text {cf }}=-1 / 4 \pi[36,37,48]$. To further explore this, we will include additional quenched disorder $a_{0}(\mathbf{x})$ coupling to $\psi^{\dagger} \psi$ that is independent of $a_{i}(\mathbf{x})$. For simplicity, we will take $a_{0}(\mathbf{x})$ to be a Gaussian random variable with zero mean and variance $W_{0}>0$. We refer to $a_{0}(\mathbf{x})$ as particle-hole violating disorder; as we will see, this terminology is justified in Dirac composite fermion mean-field theory and also in the nonlinear sigma model associated with the HLR and Dirac composite fermion theories. How such disorder $a_{0}(\mathbf{x})$ might arise if $V(\mathbf{x})$ also violates particle-hole symmetry and/or when interactions ignored in composite fermion mean-field theory are included are interesting questions we leave for future work.

Having included these effects, we can write the mean-field Lagrangian in a Dirac form by factorizing the spatial derivative terms as

$$
\begin{aligned}
\mathcal{L}_{\mathrm{HLR}}^{\mathrm{mf}}= & \psi^{\dagger}\left(i \partial_{0}+E_{F}-a_{0}\right) \psi-\psi^{\dagger} i v\left(D_{1}+i D_{2}\right) \chi \\
& -\chi^{\dagger} i v\left(D_{1}-i D_{2}\right) \psi+2 m v^{2} \chi^{\dagger} \chi
\end{aligned}
$$

using the auxiliary fermion [46]

$\chi=\frac{i}{2 m v}\left(D_{1}-i D_{2}\right) \psi, \quad \chi^{\dagger}=-\frac{i}{2 m v}\left(D_{1}^{*}+i D_{2}^{*}\right) \psi^{\dagger}$,

where $D_{j}=\partial_{j}-i a_{j}$, the Fermi energy $E_{F}>0$ fixes $\psi^{\dagger} \psi=$ $B / 4 \pi$ on average, and $a_{\mu}(\mathbf{x})$ denotes the possible quenched randomness discussed above. We set the arbitrary velocity $v>0$ to unity by rescaling $\chi$. Notice that only $\psi$ is dynamical in this Dirac formulation of HLR mean-field theory; $\chi$ is nondynamical. Introducing $\Psi=\left(\begin{array}{ll}\psi & \chi\end{array}\right)^{T}$ and the gamma matrices $\left(\Gamma^{0}, \Gamma^{1}, \Gamma^{2}\right)=\left(\sigma^{3}, i \sigma^{1}, i \sigma^{2}\right)$, where $\sigma^{i}$ are the usual Pauli matrices, the resulting mean-field Lagrangian is

$$
\begin{aligned}
\mathcal{L}_{\mathrm{HLR}}^{\mathrm{mf}}= & \bar{\Psi}(i \not \partial+\not l) \Psi-\Psi^{\dagger} P_{2}\left(i \partial_{0}+a_{0}\right) \Psi \\
& +\left(\frac{E_{F}}{2}+m\right) \Psi^{\dagger} \Psi+\left(\frac{E_{F}}{2}-m\right) \bar{\Psi} \Psi,
\end{aligned}
$$

where $\quad \bar{\Psi}=\Psi^{\dagger} \Gamma^{0}, \quad i \not \partial+\not l=\Gamma^{0}\left(i \partial_{0}+a_{0}\right)+\Gamma^{j}\left(i \partial_{j}+a_{j}\right)$, and $P_{2}=\left(\begin{array}{ll}0 & 0 \\ 0 & 1\end{array}\right)$ projects onto the second component of $\Psi$. This Lagrangian coincides with (1.1) at $\eta=1$ upon identifying $m_{1}=E_{F} / 2+m$ and $m_{2}=E_{F} / 2-m$. 


\section{B. Dirac mean-field theory}

The Dirac composite fermion Lagrangian is $[46,47,51,52]$

$$
\begin{aligned}
\mathcal{L}_{\mathrm{D}}= & \bar{\Psi}(i \not \partial+\not l) \Psi-m_{D} \bar{\Psi} \Psi-\frac{1}{4 \pi} \epsilon^{\mu \nu \rho} A_{\mu} \partial_{\nu} a_{\rho} \\
& +\frac{1}{8 \pi} \epsilon^{\mu \nu \rho} A_{\mu} \partial_{\nu} A_{\rho}+\cdots,
\end{aligned}
$$

where the Dirac notation is defined below (2.7); $a_{\mu}(t, \mathbf{x})$ is a dynamical $(2+1)$ d gauge field; $A_{\mu}(t, \mathbf{x})$ is the external electromagnetic field; $m_{D}$ is a $(2+1) \mathrm{d}$ Dirac mass; and the "..." refer to all other possible couplings and interactions that will be ignored. The Fermi velocity has been set to unity. A uniform magnetic field $\epsilon_{i j} \partial_{i} A_{j}=B>0$ and an electron filling fraction $v=1 / 2$ are assumed. Variation of $\mathcal{L}_{D}$ with respect to $A_{0}$ gives the electron density $n_{e}=\frac{B-\epsilon_{i j} \partial_{i} a_{j}}{4 \pi}$. Consequently, fluctuations about $v=1 / 2$ are controlled by $\epsilon_{i j} \partial_{i} a_{j}$. In particular, quenched electron scalar potential disorder induces randomness in the vector potential $a_{i}$. We will take this induced randomness in $a_{i}$ to be the same as in (2.4), i.e., $a_{j}$ will be a transverse Gaussian random variable with zero mean and variance $W>0$ in the mean-field approximation.

Similar to the HLR theory, the mean-field approximation consists of first imposing the $a_{0}$ equation of motion,

$$
\Psi^{\dagger} \Psi=\frac{B}{4 \pi} .
$$

Here we see that the composite fermion density is fixed, not by the electron density as is the case in the HLR theory, but by the external magnetic field. While at half-filling there is no difference between the two, differences can appear when deviations from half-filling are considered. After (2.9) is imposed, we then set all dynamical fluctuations of $a_{\mu}(t, \mathbf{x})$ to zero to achieve the mean-field approximation. In the remainder, $a_{\mu}(\mathbf{x})$ is understood to be a quenched random variable.

An important distinction between the Dirac and HLR composite fermion Lagrangians is that particle-hole symmetry has a simple implementation [70] in $\mathcal{L}_{D}$ [46,47]. In particular, particle-hole symmetry acts as time-reversal invariance in Dirac composite fermion mean-field theory: $i \rightarrow-i, t \rightarrow$ $-t, \Psi \rightarrow i \sigma^{2} \Psi$. [We see that the $P_{2}$ term in the Dirac formulation (2.7) of HLR mean-field theory explicitly violates this definition of particle-hole symmetry.] As such, the Dirac composite fermion mass $m_{D}$ breaks particle-hole symmetry. Vector potential disorder $a_{j}(\mathbf{x})$ satisfying (2.4) preserves particle-hole symmetry on average. As in the HLR theory, we consider the effects of particle-hole violating disorder by including quenched scalar potential randomness $a_{0}(\mathbf{x})$ that couples only to the top component of $\Psi$. This term explicitly violates particle-hole symmetry. We will again take $a_{0}(\mathbf{x})$ to be a Gaussian random variable with zero mean and variance $W_{0}>0$.

The resulting mean-field Lagrangian is

$$
\mathcal{L}_{D}^{\mathrm{mf}}=\bar{\Psi}(i \not \partial+\not l) \Psi-\Psi^{\dagger} P_{2} a_{0} \Psi+E_{F} \Psi^{\dagger} \Psi-m_{D} \bar{\Psi} \Psi,
$$

where $E_{F}>0$ fixes (2.9) on average and $P_{2}=\left(\begin{array}{ll}0 & 0 \\ 0 & 1\end{array}\right)$. Notice that in contrast to the HLR theory, (2.9) involves both components of $\Psi$ and so $E_{F}$ fixes the total Dirac fermion density; further comparing (2.7) with (2.10), both components of $\Psi$ are dynamical in $\mathcal{L}_{D}^{\mathrm{mf}}$. We recover the general composite-fermion mean-field Lagrangian (1.1) at $\eta=0$ upon identifying $m_{1}=$ $E_{F}$ and $m_{2}=-m_{D}$.

\section{EFFECTIVE ACTION FOR CHARGE DIFFUSION}

In this section we first construct the generating functional for disorder-averaged composite fermion Green's functions for the mean-field Lagrangian (1.1). We then derive the NLSM for composite fermion charge diffusion in the presence of particle-hole symmetric and particle-hole violating disorders.

\section{A. Generating functional}

The generating functional of disorder-averaged products of retarded/advanced composite fermion Green's functions (that determine observables such as the conductivity) is a Euclidean two-dimensional (2D) field theory. This is a 2D rather than three-dimensional (3D) field theory because, in the absence of inelastic scattering, each frequency component of the composite fermion evolves independently. Within the replica approach (see, e.g., [71,72]), this generating functional is

$$
Z=\frac{1}{\mathcal{N}} \int \mathcal{D}[\Psi] \mathcal{D}\left[\Psi^{\dagger}\right] \mathcal{D}\left[a_{\mu}\right] e^{-S},
$$

with action $S=S_{f}+S_{a}$,

$$
\begin{gathered}
S_{f}=\int d^{2} x \Psi^{\dagger}\left(\gamma^{5} \gamma^{j} D_{j}-\frac{1-\gamma^{5}}{2} a_{0}-M_{1}-M_{2} \gamma^{5}\right) \Psi \\
S_{a}=\int d^{2} x\left(\frac{1}{2 W_{0}} a_{0}^{2}+\frac{1}{2 W} a_{i}^{2}\right),
\end{gathered}
$$

and normalization $\mathcal{N}$. Here $S_{f}$ takes the form of an Euclidean $2 \mathrm{D}$ action [73] for $2 n$ Dirac fermions,

$$
\Psi_{I}=\left(\begin{array}{l}
\psi_{I} \\
\chi_{I}
\end{array}\right), \quad \Psi_{I}^{\dagger}=\left(\begin{array}{ll}
\psi_{I}^{\dagger} & \chi_{I}^{\dagger}
\end{array}\right),
$$

where $I \in\{1, \ldots, n\}$ indexes the $n$ replicas of retarded fermions and $I \in\{n+1, \ldots, 2 n\}$ indexes the $n$ replicas of advanced fermions. The gamma matrices are

$$
\gamma^{1}=\sigma^{2} \otimes \tau^{0}, \quad \gamma^{2}=\sigma^{1} \otimes \tau^{0}, \quad \gamma^{5}=-\sigma^{3} \otimes \tau^{0},
$$

where $\sigma^{j}$ are the usual Pauli matrices that act on the spinor indices and $\tau^{0}$ is the $2 n \times 2 n$ identity matrix acting on the replica indices. The replica limit $n \rightarrow 0$ is to be taken at the end of any calculation using $Z$ [74]. Up to an overall sign, $S_{f}$ coincides with $2 n$ copies of the (spatial integral of the) composite fermion mean-field Lagrangian (1.1) with the replacements $\Psi(t, \mathbf{x}) \rightarrow \Psi_{I}(\omega, \mathbf{x})$ and $i \partial_{t} \rightarrow E_{F} \pm(\omega+i \epsilon)$, where the \pm sign distinguishes between retarded/advanced fermions. The mass matrices depend on the specific composite fermion theory: in the HLR composite fermion theory,

$$
\begin{aligned}
& M_{1}=\left(\frac{E_{F}}{2}+m\right) \tau^{0}+\frac{\omega+i \epsilon}{2} \tau^{3}, \\
& M_{2}=-\left(\frac{E_{F}}{2}-m\right) \tau^{0}+\frac{\omega+i \epsilon}{2} \tau^{3} ;
\end{aligned}
$$


in the Dirac composite fermion theory,

$$
M_{1}=E_{F} \tau^{0}+(\omega+i \epsilon) \tau^{3}, \quad M_{2}=m_{D} \tau^{0} .
$$

The diagonal matrix $\tau_{I J}^{3}=\delta_{I J}$ for $I, J \in\{1, \ldots, n\}$ and $\tau_{I J}^{3}=$ $-\delta_{I J}$ for $I, J \in\{n+1, \ldots, 2 n\}$; the mass matrices are diagonal in the gamma matrix indices. The Fermi energy $E_{F}$ and frequency $\omega$ carried by $\Psi$ enter $M_{1}$ and $M_{2}$ differently in the HLR and Dirac theories because only the top component of $\Psi$ is dynamical in the HLR theory. The frequency of the composite fermion is merely a parameter in this $2 \mathrm{D}$ theory. We will set $\omega=0$ henceforth to focus on the DC conductivity. We identify

$$
m_{1}=\frac{1}{2 n} \operatorname{Tr} M_{1}, \quad m_{2}=-\frac{1}{2 n} \operatorname{Tr} M_{2} .
$$

$S_{f}$ has a $U_{R}(n) \times U_{A}(n) \subset U(2 n)$ symmetry with the infinitesimal $\epsilon>0$ distinguishing between retarded and advanced fermions.

$S_{a}$ defines the Gaussian ensembles for the independent random variables $a_{0}(\mathbf{x})$ and $a_{i}(\mathbf{x})$. The interaction between $a_{\mu}$ and $\Psi$ in this 2 D theory is responsible for the diffusion of composite fermions. Analyzing this interaction is the subject of the next section. We will assume a regularization preserving the $U(2 n)$ vector symmetry (present at $\omega=\epsilon=0$ ) that identically rotates the top $\psi=\frac{1}{2}\left(1-\gamma^{5}\right) \Psi$ and bottom $\chi=\frac{1}{2}\left(1+\gamma^{5}\right) \Psi$ spinor components of $\Psi$.

\section{B. NLSM derivation}

From the generating functional (3.1), we now derive the NLSM for composite fermion DC charge diffusion, focusing on the $\theta$ term and its physical interpretation. The derivation of the leading nontopological term in this NLSM and additional details for the calculation of the topological term can be found in Appendix B.

\section{Saddle-point analysis}

Performing the Gaussian integrals over $a_{0}$ and $a_{i}$, we obtain the "disorder-averaged action" $\bar{S}=\bar{S}^{(1)}+\bar{S}^{(2)}+\bar{S}^{(3)}$ :

$$
\begin{gathered}
\bar{S}^{(1)}=\int d^{2} x \Psi^{\dagger}\left(\gamma^{5} \gamma^{j} \partial_{j}-M_{1}-M_{2} \gamma^{5}\right) \Psi, \\
\bar{S}^{(2)}=\frac{W}{2} \int d^{2} x\left(\Psi^{\dagger} \gamma^{5} \gamma^{j} \Psi\right)\left(\Psi^{\dagger} \gamma^{5} \gamma^{j} \Psi\right), \\
\bar{S}^{(3)}=-\frac{W_{0}}{2} \int d^{2} x\left(\Psi^{\dagger} \frac{1-\gamma^{5}}{2} \Psi\right)\left(\Psi^{\dagger} \frac{1-\gamma^{5}}{2} \Psi\right) .
\end{gathered}
$$

The parentheses around fermion bilinears in $\bar{S}^{(2)}$ and $\bar{S}^{(3)}$ indicate contraction of the spinor and replica indices, e.g., $\left(\Psi^{\dagger} \gamma^{5} \gamma^{j} \Psi\right) \equiv \Psi_{a, I}^{\dagger} \gamma_{a b}^{5} \gamma_{b c}^{j} \Psi_{c, I}$ for $a, b, c \in\{1,2\}$ and $I \in$ $\{1, \ldots, 2 n\}$. The disorder-averaged action has the form of a massive $\mathrm{SU}(2 n)$ Thirring model [26] (with coupling $-W$ ) plus an additional chiral interaction $\bar{S}^{(3)}$. This interacting Euclidean 2D theory is translated to Minkowski signature in Appendix A.

We perform a semiclassical analysis of the interactions in $\bar{S}$. To this end, we first rewrite $\bar{S}^{(2)}$ as

$$
\bar{S}^{(2)}=\frac{W}{2} \int d^{2} x\left[\left(\Psi_{I}^{\dagger} \Psi_{J}\right)\left(\Psi_{J}^{\dagger} \Psi_{I}\right)-\left(\Psi_{I}^{\dagger} \gamma^{5} \Psi_{J}\right)\left(\Psi_{J}^{\dagger} \gamma^{5} \Psi_{I}\right)\right]
$$

and then we decouple each of the interactions using the Hubbard-Stratonovich fields $X_{I J}, Y_{I J}$, and $Z_{I J}$ as

$$
e^{-\bar{S}^{(2)}-\bar{S}^{(3)}}=\int \mathcal{D}[X] \mathcal{D}[Y] \mathcal{D}[Z] e^{-\int d^{2} x\left[\frac{1}{2 W} \operatorname{Tr}\left(X^{2}+Y^{2}\right)+\frac{1}{2 W_{0}} \operatorname{Tr} Z^{2}-i\left(X_{I J}+\frac{Z_{I J}}{2}\right) \Psi_{J}^{\dagger} \Psi_{I}-\left(Y_{I J}-i \frac{Z_{I J}}{2}\right) \Psi_{J}^{\dagger} \gamma^{5} \Psi_{I}\right]},
$$

where $\operatorname{Tr} X^{2} \equiv X_{J I} X_{I J}, \operatorname{Tr} Y^{2} \equiv Y_{J I} Y_{I J}$, and $\operatorname{Tr} Z^{2} \equiv Z_{J I} Z_{I J}$. Each of the fields $X_{I J}, Y_{I J}$, and $Z_{I J}$ transform in the adjoint rep of $U(2 n)$. Next, we look for solutions to the saddle-point equations (at $\epsilon=0)$ for $\left\langle X_{I J}\right\rangle,\left\langle Y_{I J}\right\rangle$, and $\left\langle Z_{I J}\right\rangle$ :

$$
\begin{aligned}
& X=\frac{W}{2} \operatorname{Tr}_{\sigma}\left(\int \frac{d^{2} k}{(2 \pi)^{2}} \frac{i}{i \gamma^{5} \gamma^{j} k_{j}-\left(M_{1}+i X+i \frac{Z}{2}\right)-\left(M_{2}+Y-i \frac{Z}{2}\right) \gamma^{5}}\right), \\
& Y=\frac{W}{2} \operatorname{Tr}_{\sigma}\left(\int \frac{d^{2} k}{(2 \pi)^{2}} \frac{\gamma^{5}}{i \gamma^{5} \gamma^{j} k_{j}-\left(M_{1}+i X+i \frac{Z}{2}\right)-\left(M_{2}+Y-i \frac{Z}{2}\right) \gamma^{5}}\right), \\
& Z=\frac{W_{0}}{4} \operatorname{Tr}_{\sigma}\left(\int \frac{d^{2} k}{(2 \pi)^{2}} \frac{1-\gamma^{5}}{i \gamma^{5} \gamma^{j} k_{j}-\left(M_{1}+i X+i \frac{Z}{2}\right)-\left(M_{2}+Y-i \frac{Z}{2}\right) \gamma^{5}}\right),
\end{aligned}
$$

where the trace $\operatorname{Tr}_{\sigma}$ is only taken over the spinor indices; and we write $X$ for $\left\langle X_{I J}\right\rangle$ and similarly for $Y$ and $Z$. We consider the $U_{R}(n) \times U_{A}(n)$-preserving ansatz:

$$
\begin{gathered}
\langle X\rangle=\left(\Gamma_{+}+\Gamma_{-}\right) \tau^{3}+i X_{0} \tau^{0}, \\
\langle Y\rangle=Y_{0} \tau^{0}, \\
\langle Z\rangle=-2 \Gamma_{-} \tau^{3}+i Z_{0} \tau^{0},
\end{gathered}
$$

where $\Gamma_{ \pm}, X_{0}, Y_{0}$, and $Z_{0}$ are real. $\Gamma_{ \pm}$parametrize nonzero scattering rates of the composite fermions, while $X_{0}, Y_{0}$, and $Z_{0}$ parametrize possible renormalization of $m_{1}$ and $m_{2}$ [recall Eq. (3.8)]. We have set to zero a possible term in the ansatz for $\langle Y\rangle$ proportional to $\tau^{3}$ that appears to prevent a $U_{R}(n) \times U_{A}(n)$-preserving solution to these equations; preliminary analysis indicates that such a term requires a more general ansatz that only preserves a subgroup of $U_{R}(n) \times U_{A}(n)$. 
We next present the detailed solution to these equations that results from the ansatz above. The conclusion from this analysis will be that the composite fermions obtain the renormalized mass matrix

$$
\left(\begin{array}{cc}
\left(m_{1}^{R}+m_{2}^{R}\right) \tau^{0}+i\left(\Gamma_{+}^{R}-\Gamma_{-}^{R}\right) \tau^{3} & 0 \\
0 & \left(m_{1}^{R}-m_{2}^{R}\right) \tau^{0}+i\left(\Gamma_{+}^{R}+\Gamma_{-}^{R}\right) \tau^{3}
\end{array}\right) .
$$

Here the $R$ superscript (dropped in the next section) indicates renormalized parameters. We will find that the saddle-point solutions allow nonzero $m_{2}^{R}$ and unequal scattering rates $\Gamma_{-}^{R} \neq 0$ for the two components of $\Psi$ only when particle-hole violating disorder is present $\left(W_{0} \neq 0\right)$.

To this end, we begin by defining the real parameters $J_{1}$ and $J_{2}$ :

$$
i J_{1} \tau^{3}+J_{2} \tau^{0} \equiv \int \frac{d^{2} k}{(2 \pi)^{2}} \frac{1}{k^{2}+\left(M_{1}+i X+i \frac{Z}{2}\right)^{2}+\left(M_{2}+Y-i \frac{Z}{2}\right)^{2}} .
$$

By direct evaluation we find

$$
\begin{gathered}
J_{1}=\frac{\operatorname{sgn}\left(\Gamma_{-} \tilde{m}_{2}-\Gamma_{+} \tilde{m}_{1}\right)}{8 \pi}\left[\pi-2 \arctan \left(\frac{\tilde{m}_{2}^{2}-\tilde{m}_{1}^{2}-\Gamma_{-}^{2}+\Gamma_{+}^{2}}{2\left|\Gamma_{-} \tilde{m}_{2}-\Gamma_{+} \tilde{m}_{1}\right|}\right)\right], \\
J_{2}=-\frac{1}{8 \pi} \log \left(\frac{\Lambda^{4}}{\left[\left(\Gamma_{+}+\Gamma_{-}\right)^{2}+\left(\tilde{m}_{1}+\tilde{m}_{2}\right)^{2}\right]\left[\left(\Gamma_{+}-\Gamma_{-}\right)^{2}+\left(\tilde{m}_{1}-\tilde{m}_{2}\right)^{2}\right]}\right),
\end{gathered}
$$

Here we have introduced the shifted masses

$$
\begin{gathered}
\tilde{m}_{1}=m_{1}-X_{0}-Z_{0}, \\
\tilde{m}_{2}=-m_{2}-Y_{0},
\end{gathered}
$$

and UV cutoff $\Lambda$. We search for a self-consistent solution when $\left|\Gamma_{ \pm} / \tilde{m}_{1}\right| \ll 1$ and $\left|\tilde{m}_{2} / \tilde{m}_{1}\right| \ll 1$ : this corresponds to the small scattering rate limit $\Gamma_{ \pm} / E_{F} \ll 1$ with approximate particle-hole symmetry. In this regime, $J_{1} \approx-1 / 4 \pi$ and $J_{2} \approx$ $\frac{1}{2 \pi} \log \left(\left|\tilde{m}_{1}\right| / \Lambda\right)$. Written in terms of these parameters the saddle-point equations (3.14)-(3.16) become

$$
\begin{gathered}
2 \Gamma_{-}=W_{0}\left[J_{1}\left(\tilde{m}_{1}+\tilde{m}_{2}\right)+J_{2}\left(\Gamma_{+}+\Gamma_{-}\right)\right], \\
Z_{0}=-W_{0}\left[J_{1}\left(\Gamma_{+}+\Gamma_{-}\right)-J_{2}\left(\tilde{m}_{1}+\tilde{m}_{2}\right)\right], \\
\Gamma_{+}+\Gamma_{-}=-W\left(J_{1} \tilde{m}_{1}+J_{2} \Gamma_{+}\right), \\
X_{0}=-W\left(J_{1} \Gamma_{+}-J_{2} \tilde{m}_{1}\right), \\
0=W\left(J_{1} \tilde{m}_{2}+J_{2} \Gamma_{-}\right), \\
Y_{0}=-W\left(J_{1} \Gamma_{-}+J_{2} \tilde{m}_{2}\right) .
\end{gathered}
$$

We solve these equations for $X_{0}, Y_{0}, Z_{0}, \Gamma_{ \pm}$, and $m_{2}$ :

$$
\begin{gathered}
m_{2}=\frac{m_{1} W_{0}\left[J_{2}+W\left(J_{1}^{2}+J_{2}^{2}\right)\right]}{2+J_{2}\left(3 W_{0}+4 W\right)+W\left(3 W_{0}+2 W\right)\left(J_{1}^{2}+J_{2}^{2}\right)}, \\
Y_{0}=\frac{-m_{1} W_{0} W\left(J_{1}^{2}+J_{2}^{2}\right)}{2+J_{2}\left(3 W_{0}+4 W\right)+W\left(3 W_{0}+2 W\right)\left(J_{1}^{2}+J_{2}^{2}\right)}, \\
\Gamma_{-}=\frac{m_{1} W_{0} J_{1}}{2+J_{2}\left(3 W_{0}+4 W\right)+W\left(3 W_{0}+2 W\right)\left(J_{1}^{2}+J_{2}^{2}\right)}, \\
\Gamma_{+}+\Gamma_{-}=\frac{-2 m_{1} W J_{1}}{2+J_{2}\left(3 W_{0}+4 W\right)+W\left(3 W_{0}+2 W\right)\left(J_{1}^{2}+J_{2}^{2}\right)}, \\
Z_{0}=\frac{2 m_{1} W_{0}\left(J_{2}+W\left(J_{1}^{2}+J_{2}^{2}\right)\right)}{2+J_{2}\left(3 W_{0}+4 W\right)+W\left(3 W_{0}+2 W\right)\left(J_{1}^{2}+J_{2}^{2}\right)}, \\
X_{0}=\frac{m_{1} W\left[2 J_{2}+\left(W_{0}+2 W\right)\left(J_{1}^{2}+J_{2}^{2}\right)\right]}{2+J_{2}\left(3 W_{0}+4 W\right)+W\left(3 W_{0}+2 W\right)\left(J_{1}^{2}+J_{2}^{2}\right)} .
\end{gathered}
$$

Notice that $Y_{0}, \Gamma_{-}, Z_{0}$, and $m_{2}$ vanish as $W_{0} \rightarrow 0$. We treat $m_{2}$ as a variable in order to avoid an overly constrained set of equations; our interpretation is that $W_{0}$ and $W$ disorders cause $m_{2}$ to renormalize to a value determined by the saddle-point 
equations. To ensure the scattering rates $\Gamma_{+} \pm \Gamma_{-} \geqslant 0$ of $\frac{1 \pm \gamma^{5}}{2} \Psi$ are nonnegative, we require

$$
J_{1}\left[2+J_{2}\left(3 W_{0}+4 W\right)+W\left(3 W_{0}+2 W\right)\left(J_{1}^{2}+J_{2}^{2}\right)\right] \leqslant 0 .
$$

The retarded/advanced 2D Euclidean fermion Green's function that obtains from this saddle-point solution is

$$
\bar{g}_{R / A}\left(\omega, \mathbf{x}, \mathbf{x}^{\prime}\right)=\left\langle\mathbf{x}\left|\frac{1}{i \sigma_{1} \partial_{1}-i \sigma_{2} \partial_{2}+\left(m_{1}-X_{0}-Z_{0}+\omega \pm i \Gamma_{+}\right)+\left(m_{2}+Y_{0}+\omega \mp i \Gamma_{-}\right) \sigma_{3}}\right| \mathbf{x}^{\prime}\right\rangle .
$$

We have included nonzero $\omega$, parametrizing deviations about the Fermi energy. This Green's function suggests how to absorb the logarithmic divergence $J_{2}$ into renormalized parameters. We first introduce the renormalization factors,

$$
\begin{gathered}
Z_{R} \equiv \frac{2+J_{2}\left(3 W_{0}+4 W\right)+W\left(3 W_{0}+2 W\right)\left(J_{1}^{2}+J_{2}^{2}\right)}{\left(2+W_{0} J_{2}+2 W J_{2}\right)}, \\
Z_{L} \equiv \frac{J_{2}+W\left(J_{1}^{2}+J_{2}^{2}\right)}{J_{2}} .
\end{gathered}
$$

Then the renormalized $m_{1,2}$ and $\Gamma_{ \pm}$are

$$
\begin{gathered}
m_{1}^{R}=m_{1}-X_{0}-Z_{0}=\frac{m_{1}}{Z_{R}}, \\
m_{2}^{R}=m_{2}+Y_{0}=\frac{m_{2}}{Z_{L}}, \\
\Gamma_{-}^{R}=W_{0} \frac{m_{1}^{R} J_{1}}{2+\left(W_{0}+2 W\right) J_{2}}, \\
\Gamma_{+}^{R}=-\left(W_{0}+2 W\right) \frac{m_{1}^{R} J_{1}}{2+\left(W_{0}+2 W\right) J_{2}} .
\end{gathered}
$$

The condition (3.38) ensures the renormalized $\Gamma_{+}^{R} \pm \Gamma_{-}^{R}$ are nonnegative. Notice that $X_{0}$ and $Z_{0}$ are absorbed into a renormalization of $m_{1}$, while $Y_{0}$ is a renormalization of $m_{2}$. We use these renormalized parameters in the remainder without the $R$ superscript to determine the $\theta$ angle.

\section{2. $\theta$ term}

The saddle-point solutions for $\langle X\rangle$ and $\langle Z\rangle$-in particular, the nonzero scattering rates $\Gamma_{ \pm}$-spontaneously break the $U(2 n)$ symmetry (present at $\epsilon=0$ ) to $U(n)_{R} \times U(n)_{A}$. We consider the fluctuations about this saddle-point by writing $Q_{1}(\mathbf{x}) \equiv[X(\mathbf{x})+Z(\mathbf{x})] /\left(\Gamma_{+}-\Gamma_{-}\right)=U_{1}(\mathbf{x}) \tau^{3} U_{1}^{\dagger}(\mathbf{x})$ and $\quad Q_{2}(\mathbf{x}) \equiv X(\mathbf{x}) /\left(\Gamma_{+}+\Gamma_{-}\right)=U_{2}(\mathbf{x}) \tau^{3} U_{2}^{\dagger}(\mathbf{x}) \quad$ where $U_{1}(\mathbf{x}), U_{2}(\mathbf{x}) \in U(2 n)$. This parametrization ensures that $Q_{1,2}(\mathbf{x}) \quad$ satisfy $Q_{1,2}^{2}(\mathbf{x})=1$. Since $Q_{1,2}(\mathbf{x})=\tau^{3}$ for $U_{1,2}(\mathbf{x}) \in U_{R}(n) \times U_{A}(n)$, the target manifold of $Q_{1,2}(\mathbf{x})$ is $U(2 n) / U_{R}(n) \times U_{A}(n)$. Recall that $\langle Y\rangle$ has been absorbed into a renormalized $m_{2}$ after which $\langle Y\rangle=0$. Consequently, fluctuations about $\langle Y\rangle$ are massive and will not be considered further. Since the action is only invariant under global vector $U(2 n)$ rotations (under which $\frac{1-\gamma^{5}}{2} \Psi$ and $\frac{1+\gamma^{5}}{2} \Psi$ transform identically), the gapless Goldstone bosons correspond to those fluctuations of $Q_{1}$ and $Q_{2}$ for which $U_{1}=U_{2}$; the "axial" fluctuations $Q_{1} \neq Q_{2}$ are massive (see Appendix B 2 for an explicit demonstration) and can be neglected at low energies. Thus, we have a single light matrix boson $Q=Q_{1}=Q_{2} \in U(2 n) / U_{R}(n) \times U_{A}(n)$ that we parametrize as $Q(\mathbf{x})=U(\mathbf{x}) \tau^{3} U^{\dagger}(\mathbf{x})$ with $U(\mathbf{x}) \in U(2 n)$.
The NLSM (1.2) for $Q$ is obtained by integrating out the fermions,

$e^{-S_{\mathrm{NLSM}}}=\int \mathcal{D} \Psi \mathcal{D} \Psi^{\dagger} e^{-\int d^{2} x \Psi^{\dagger}\left[\gamma^{5} \gamma^{j} \partial_{j}-\left(M_{1}+i \Gamma_{+} Q\right)-\left(M_{2}+i \Gamma_{-} Q\right) \gamma^{5}\right] \Psi}$.

The real part of $S_{\mathrm{NLSM}}$, which describes the longitudinal conductivity of the system $1 / g \propto W \gg 1$, is calculated in Appendix B 3. The imaginary part of $S_{\mathrm{NLSM}}$ is the topological $\theta$ term,

$$
S_{\text {top }}=i \frac{\theta}{16 \pi} \int d^{2} x \operatorname{Tr}\left(\epsilon_{i j} Q \partial_{i} Q \partial_{j} Q\right) .
$$

This term weights $Q$ configurations by the second homotopy group $\Pi_{2}\left[U(2 n) / U_{R}(n) \times U_{A}(n)\right]=\mathbb{Z}$. Since this classification is independent of the replica index for any positive integer $n$, we set $n=1$ and so $Q(\mathbf{x})=$ $U(\mathbf{x}) \tau^{3} U^{\dagger}(\mathbf{x}) \in U(2) / U_{R}(1) \times U_{A}(1)=\mathrm{SU}(2) / U(1) \quad$ with $U(\mathbf{x}) \in \mathrm{SU}(2)$. Furthermore, since the classification of $Q$ configurations by $\mathbb{Z}$ arises from the relative $U(1)$ subgroup of $U_{R}(n) \times U_{A}(n)$, the result we find for $\theta$ should continue to hold in the replica limit $n \rightarrow 0$ [58].

To extract the topological term, it is convenient to perform the gauge transformation $\Psi \rightarrow U(\mathbf{x}) \Psi$ before integrating out the fermions. This allows us to interpret the fluctuations of $Q$ in terms of the $\mathrm{SU}(2)$ gauge field,

$$
A_{j}=i U^{\dagger} \partial_{j} U
$$

(Note that $A_{j}$ here and below is not the electromagnetic vector potential, despite our use of the same symbol.) Thus, we compute

$$
e^{-S_{\mathrm{NLSM}}}=\int \mathcal{D} \Psi \mathcal{D} \Psi^{\dagger} e^{-\int d^{2} x \Psi^{\dagger}\left[\gamma^{5} \gamma^{j}\left(\partial_{j}-i A_{j}\right)-\varphi_{1}-\varphi_{2} \gamma^{5}\right] \Psi},
$$

where

$$
\varphi_{1}=m_{1} \tau^{0}+i \Gamma_{+} \tau^{3}, \quad \varphi_{2}=-m_{2} \tau^{0}+i \Gamma_{-} \tau^{3} .
$$

It is sufficient to determine $S_{\mathrm{NLSM}}$ to quadratic order in $A_{i}$ to obtain the topological term, which is cubic in $Q$. Using (3.48) to relate $Q$ to $A_{j}$ and the identity $\epsilon_{j k} \partial_{j} A_{k}=i \epsilon_{j k} A_{j} A_{k}$ for a pure gauge potential, the topological $\theta$ term

$$
S_{\text {top }}=\frac{1}{4 \pi} \int d^{2} x \operatorname{Tr}\left[\tau^{3} \epsilon_{j k}\left(\theta^{\mathrm{I}} \partial_{j} A_{k}+i \theta^{\mathrm{II}} A_{j} A_{k}\right)\right]
$$

and the $\theta$ angle

$$
\theta=\theta^{\mathrm{I}}+\theta^{\mathrm{II}} .
$$

$\theta^{\mathrm{II}}$ and $\theta^{\mathrm{I}}$ are associated with the "classical" and "quantum" contributions to the Hall conductivity of the system [75]. 
We will now calculate each of these contributions to $\theta$ (see Appendix B 4 for additional details). We will combine a result of Goldstone and Wilczek [76], familiar from work on topological insulators [77], with a direct evaluation; a similar argument in this context can be found in [61].

Because $\theta^{\mathrm{I}}$ in (3.51) is only sensitive to the Abelian subgroup of SU(2) generated by $\tau^{3}$, we can simplify its determination by focusing on the associated Abelian gauge field $\frac{1}{2} \operatorname{Tr}\left(\tau^{3} A_{j}\right)$ under which the retarded and advanced components of $\Psi$ carry opposite charge. We will furthermore treat $\varphi_{1}$ and $\varphi_{2}$ in Eq. (3.50) as smoothly varying complex fields that assume their fixed values at the end of this calculation. Writing $\varphi_{1,2}$ in terms of the complex fields $\chi_{1,2}^{R, A}$,

$\varphi_{1}+\varphi_{2}=\left(\begin{array}{cc}e^{i \chi_{1}^{R}} & 0 \\ 0 & e^{i \chi_{1}^{A}}\end{array}\right), \quad \varphi_{1}-\varphi_{2}=-\left(\begin{array}{cc}e^{-i \chi_{2}^{R}} & 0 \\ 0 & e^{-i \chi_{2}^{A}}\end{array}\right)$,

the generalization of [76] to complex $\varphi_{1,2}$ gives the linear in $A_{j}$ contribution to $S_{\text {top }}$ :

$$
S_{\text {top }}^{\mathrm{I}}=\frac{1}{8 \pi} \int d^{2} x \epsilon_{j k} \partial_{j}\left(\chi_{1}^{R}+\chi_{2}^{R}-\chi_{1}^{A}-\chi_{2}^{A}\right) \operatorname{Tr}\left(\tau^{3} A_{k}\right) .
$$

After an integration by parts, we identify

$$
\theta^{\mathrm{I}}=-\frac{1}{2}\left(\chi_{1}^{R}+\chi_{2}^{R}-\chi_{1}^{A}-\chi_{2}^{A}\right) .
$$

We now evaluate $\theta^{\mathrm{I}}$ on the saddle-point solution by setting $\varphi_{1}$ and $\varphi_{2}$ to their values in (3.50). To ensure that $\theta^{\mathrm{I}}$ is well defined modulo an integer multiple of $2 \pi$, we constrain the real parts $\operatorname{Re}\left[\chi_{1,2}^{R, A}\right] \in[0, \pi)$. For general $\varphi_{1,2}$ it is necessary to perform some combination of a charge conjugation,

$$
\mathcal{C} \Psi \mathcal{C}=\gamma^{2} \Psi^{*},
$$

and a chiral rotation with $\alpha=\pi / 2$ [see (A7)] on each fermion species in order to solve for $\chi_{1,2}^{R, A}$. Note that a flip of the relative sign in $\chi_{1}^{R}+\chi_{2}^{R}-\chi_{1}^{A}-\chi_{2}^{A}$ accompanies a charge conjugation on an advanced or retarded fermion. Likewise, any chiral rotation would generally include an additional contribution to the NLSM action due to the anomalous variation of the fermion measure [78] in Eq. (3.49). For the $\varphi_{1,2}$ under consideration, it is only necessary to perform a charge conjugation on the advanced fermions with the result (see Appendix B 4 b)

$$
\theta^{\mathrm{I}}=\pi+\arctan \left(\frac{\Gamma_{+}-\Gamma_{-}}{m_{1}+m_{2}}\right)-\arctan \left(\frac{\Gamma_{+}+\Gamma_{-}}{m_{1}-m_{2}}\right) .
$$

In the diffusive regime $0<\Gamma_{1,2} \ll E_{F}$ with weak particlehole symmetry violation $\left|\Gamma_{-}\right| \propto W_{0} \ll W$,

$$
\theta^{\mathrm{I}}=\pi+O\left(W_{0}\right) .
$$

Equation (3.51) indicates that $\theta^{\mathrm{II}}$ is sensitive to the non-Abelian nature of $\mathrm{SU}(2)$. By direct evaluation of (3.49) of the quadratic in $A_{j}$ contribution to $S_{\text {top }}$, we find (see Appendix B 4 a)

$$
\begin{aligned}
\theta^{\mathrm{II}}=\frac{m_{1} \Gamma_{-}+m_{2} \Gamma_{+}}{m_{1} \Gamma_{+}+m_{2} \Gamma_{-}} & \arctan \left(\frac{\Gamma_{+}-\Gamma_{-}}{m_{1}+m_{2}}\right) \\
& \left.+\arctan \left(\frac{\Gamma_{+}+\Gamma_{-}}{m_{1}-m_{2}}\right)\right] .
\end{aligned}
$$

Notice that $\theta^{\mathrm{II}} \neq 0$ only when particle-hole violating disorder $W_{0} \neq 0$ is present. The $\theta$ term only receives a contribution from $\theta^{\mathrm{I}}$ when the disorder is particle-hole symmetric because the particle-hole violating mass $m_{2}$ vanishes in the saddlepoint solution.

\section{DISCUSSION}

In this article we studied the effect of particle-hole symmetry violation on the integer quantum Hall plateau transition using the HLR and Dirac composite fermion mean-field theories. For the particle-hole violating perturbations we considered, we showed that the diffusive quantum criticality of this transition is described by the same nonlinear sigma model (NLSM). The derivation of this NLSM proceeded by a semiclassical analysis in which Schwinger-Dyson equations, associated with the "interactions" produced by the various types of quenched disorders in the $(2+0)$ d generating functional of composite fermion Green's functions (3.1), are solved via a particular ansatz. (Here we used the replica trick to obtain this generating functional with $2 n$ replicas.) Our ansatz preserved the $U_{R}(n) \times U_{A}(n)$ symmetry present in this generating functional. For particle-hole symmetric quenched disorder (see Sec. II A for the definition), we found that the solution to the Schwinger-Dyson equations-in particular, finite composite fermion DC conductivity $\sigma_{x x}^{\text {cf }}$-required the vanishing of a particle-hole breaking (renormalized) mass term corresponding to $m_{2}$ in (1.1). In the Dirac composite fermion theory, this term is the usual $(2+1) \mathrm{d}$ Dirac mass; in the HLR theory, this mass term is controlled by the difference of the renormalized Fermi energy and effective mass. The resulting NLSM contains a topological $\theta=\pi$ term. This suggests an emergent particle-hole symmetry of the diffusive quantum critical point. It would be interesting to explore other ansatze, e.g., those associated with flag manifolds [79], with reduced symmetry to better understand this interpretation.

We also considered the effect of particle-hole symmetry breaking quenched disorder. We found such disorder can shift the $\theta$ angle away from $\pi$. This corresponds to a violation of particle-hole symmetric electrical transport. Because the NLSM that we study is only appropriate for longitudinal conductivities $\sigma_{x x}^{\text {cf }} \gg 1$ (in units of $e^{2} / h$ ), we are not able to determine the identity of the state that obtains for $\sigma_{x x}^{\text {cf }} \sim 1$ towards which the nonlinear sigma model evolves at low energies. [Recall that the longitudinal conductivity is a coupling in the nonlinear sigma model: in the absence of a topological $\theta$ term, $\sigma_{x x}^{\text {cf }} \rightarrow 0$ in the IR; with a topological $\theta$ term, it is expected that $\sigma_{x x}^{\text {cf }}$ takes an $O(1)$ fixed point value.] It would be interesting to understand if particle-hole symmetry emerges for $\sigma_{x x}^{\mathrm{cf}} \sim 1$, as predicted in [80] and found in a numerical study of noninteracting electrons [81]. Alternatively, if particle-hole symmetry does not emerge, we expect either a gapped insulator or a diffusive metal (at least in the vicinity 
of the particle-hole symmetric limit at $\theta=\pi$ ). It would be interesting to connect our result with recent work [32] that found evidence for a line of extended states with continuously varying critical exponents as particle-hole symmetry is violated by varying the coefficient of electron scalar potential in Eq. (2.3).

In [54] the $\theta=\pi$ term was extracted by a careful study of the chiral anomaly of a 2D theory closely related to the one in (3.49). In this earlier study, only particle-hole symmetric disorder was considered. We have been unsuccessful in applying this chiral anomaly argument to the case studied in this paper, where particle-hole violating disorder is included. The technical issue is that a nonunitary chiral rotation is required to relate the retarded and advanced fermion mass matrices. We are unaware how the gauge field effective action changes under such transformations. Perhaps the methods in [82] can shed light on this question.

\section{ACKNOWLEDGMENTS}

We thank S. Raghu for useful conversations and correspondence. This material is based upon work supported by the U.S. Department of Energy, Office of Science, Office of Basic Energy Sciences under Award No. DE-SC0020007. M.M. acknowledges the generous hospitality of the Stanford Institute for Theoretical Physics and support provided by the Moore Foundation for this work. P.K. is supported by DOE-BES Grant No. DE-SC0002140.

\section{APPENDIX A: MINKOWSKI ACTION}

In this Appendix we translate the 2D Euclidean disorderaveraged action $\bar{S}=\bar{S}^{(1)}+\bar{S}^{(2)}+\bar{S}^{(3)}$ in (3.9)-(3.11) into Minkowski signature. We first define the Minkowski spinors [73],

$$
\Phi=e^{\frac{\pi}{4} \gamma^{2} \gamma^{5}} \Psi, \quad \Phi^{\dagger}=\Psi^{\dagger} e^{\frac{\pi}{4} \gamma^{2} \gamma^{5}} .
$$

Note the same transformation is used for $\Phi$ and $\Phi^{\dagger}$. The Euclidean-signature gamma matrices are given in (3.5). Next we introduce the Minkowski-signature $(+1,-1)$ gamma matrices,

$$
\Gamma^{0}=-\gamma^{5}, \quad \Gamma^{1}=i \gamma^{1}, \quad \Gamma^{5}=i \Gamma^{0} \Gamma^{1}=i \gamma^{2},
$$

and Wick rotate $x^{0}=-i x^{2}, x^{1}=x^{1}$. [We caution that we are redefining in this Appendix the uppercase gamma matrices previously employed in the $(2+1) \mathrm{d}$ composite fermion mean-field theories in Eqs. (2.7) and (2.10).] Making these replacements in Eqs. (3.9) and (3.10), we find the Minkowskisignature action $\bar{S}_{M}=\bar{S}_{M}^{(1)}+\bar{S}_{M}^{(2)}+\bar{S}_{M}^{(3)}$ :

$$
\begin{array}{r}
\bar{S}_{M}^{(1)}=\int d x^{0} d x^{1} \bar{\Phi}\left[i \Gamma^{\mu} \partial_{\mu}+M\left(\frac{1+\Gamma^{5}}{2}\right)\right. \\
\left.+M^{*}\left(\frac{1-\Gamma^{5}}{2}\right)\right] \Phi, \\
\bar{S}_{M}^{(2)}=\frac{W}{2} \int d x^{0} d x^{1}\left(\bar{\Phi} \Gamma^{\mu} \Phi\right)\left(\bar{\Phi} \Gamma_{\mu} \Phi\right), \\
\bar{S}_{M}^{(3)}=-\frac{W_{0}}{2} \int d x^{0} d x^{1}\left(\bar{\Phi} \frac{1+i \Gamma^{5}}{2} \Phi\right)^{2},
\end{array}
$$

where $\mu \in\{0,1\}, \bar{\Phi}=\Phi^{\dagger} \Gamma^{0}$, and the complex mass matrix $M=M_{2}-i M_{1}$ with the mass matrices for the HLR and Dirac theories given in Eqs. (3.6) and (3.7). We see that $M_{2}$ is a conventional Dirac mass and $M_{1}$ is an axial mass. $\bar{S}_{M}^{(2)}$ is a $\mathrm{SU}(2 n)$ Thirring interaction with coupling $-W$; the chiral interaction $\bar{S}_{M}^{(3)}$ is a chiral repulsion $W_{0}>0$.

In Minkowski signature, the chiral transformation is

$$
\Phi \rightarrow e^{i \alpha \Gamma^{5}} \Phi, \quad \Phi^{\dagger} \rightarrow \Phi^{\dagger} e^{-i \alpha \Gamma^{5}},
$$

where $\alpha$ is a real. Using (A1) and (A6), the Euclideansignature fermions transform under a chiral transformation as

$$
\Psi \rightarrow e^{i \alpha \gamma^{5}} \Psi, \quad \Psi^{\dagger} \rightarrow \Psi^{\dagger} e^{i \alpha \gamma^{5}} .
$$

Notice again that Hermitian conjugation and Wick rotation (between Euclidean and Minkowski signatures) do not commute. The derivative terms and Thirring interaction are invariant under continuous chiral rotations; the mass terms and interaction proportional to $W_{0}$ are only invariant under the $\mathbb{Z} / 2$ subgroup for which $\alpha \in \pi \mathbb{Z}$.

\section{APPENDIX B: DETAILED NLSM DERIVATION}

In this Appendix we detail the calculation of the NLSM for composite fermion mean-field theory that is sketched in Sec. III B.

\section{Setup}

Here we have found it convenient to employ a different convention than the one used in the main text. The translation table between the two conventions is given below:

$$
\begin{gathered}
\Gamma_{+}=\frac{\alpha_{2}}{2}, \\
\Gamma_{-}=\frac{-\alpha_{1}}{2}, \\
\gamma_{1}=\sigma_{y}, \quad \gamma_{2}=\sigma_{x}, \quad \gamma_{5}=-\sigma_{z} .
\end{gathered}
$$

Above $\left(\sigma_{x}, \sigma_{y}, \sigma_{z}\right)$ are the usual Pauli sigma matrices that act on the spinor indices; note that we are leaving implicit the replica indices since the gamma matrices are diagonal in replica space [see (3.5)]. Furthermore, we reflect both coordinates $(x, y) \rightarrow$ 
$(-x,-y)$ and reverse the overall signs of the fermion and NLSM actions in (3.49):

$$
e^{S_{\mathrm{NLSM}}}=\int \mathcal{D} \Psi \mathcal{D} \Psi^{\dagger} e^{S}
$$

with

$$
S=\int d^{2} x \Psi^{\dagger}\left(i \sigma_{x} \partial_{x}-i \sigma_{y} \partial_{y}-M_{2} \sigma_{z}+M_{1} \sigma_{0}+i \frac{\alpha_{1} \sigma_{z}+\alpha_{2} \sigma_{0}}{2} Q\right) \Psi .
$$

Here $\sigma_{0}$ is the $2 \times 2$ identity matrix. The masses $M_{1}$ and $M_{2}$ for the HLR and Dirac theories are given in (3.6) and (3.7); we set $\omega=\epsilon=0$ in these expressions for the masses. The matrix boson $Q(\mathbf{x})=U(\mathbf{x}) \tau^{3} U^{\dagger}(\mathbf{x})$ for $U(\mathbf{x}) \in U(2 n)$ and $\tau^{3}=\sigma_{z} \otimes \sigma_{0}^{n}$. Since all terms except for $Q$ are singlets with respect to the replica indices (i.e., the $\tau$ space), we (generally) leave implicit the $2 n \times 2 n$ identity matrix $\tau_{0}$ that acts in this subspace.

We partially follow [61] to separately derive the real and imaginary parts of $S_{\mathrm{NLSM}}$. Before proceeding, we define the selfconsistent Born approximation (SCBA) Green's functions,

$$
g_{ \pm} \equiv \frac{1}{M_{1} \sigma_{0}+i \sigma_{x} \partial_{x}-i \sigma_{y} \partial_{y}-M_{2} \sigma_{z} \pm i\left(\frac{\alpha_{1} \sigma_{z}+\alpha_{2} \sigma_{0}}{2}\right) \tau^{3}},
$$

which are related to the retarded and advanced Green's functions $g_{R, A}$ as follows:

$$
\begin{gathered}
\left(g_{+}\right)^{\dagger}=g_{-}, \quad g_{+}=\operatorname{Diag}\left(g_{R}, g_{A}\right)_{\tau}, \quad g_{-}=\operatorname{Diag}\left(g_{A}, g_{R}\right)_{\tau}, \\
g_{+}-g_{-}=\left(g_{R}-g_{A}\right) \tau^{3}, \quad g_{+}+g_{-}=\left(g_{R}+g_{A}\right) \tau_{0} .
\end{gathered}
$$

Note that the \pm subscript of $g_{ \pm}$labels the sign of the imaginary part of the Green's function.

\section{Goldstone parametrization}

In the main text we argued that the massless Goldstone modes correspond to $Q_{1}=Q_{2}$ fluctuations, where the matrix bosons $Q_{1}, Q_{2} \in U(2 n) / U(n) \times U(n)$. The $Q_{1}=Q_{2}$ fluctuations correspond to vector gauge transformations of $\Psi$. Here we show explicitly that axial gauge transformations corresponding to $Q_{1} \neq Q_{2}$ fluctuations are massive.

Consider the two-field sigma model involving $Q_{1}$ and $Q_{2}$ :

$$
S\left[Q_{1}, Q_{2}\right] \equiv \operatorname{Tr} \ln \left[\left(M_{1} \sigma_{0}-M_{2} \sigma_{z}+i \sigma_{x} \partial_{x}-i \sigma_{y} \partial_{y}\right)+\left(\begin{array}{cc}
i \Gamma_{1} Q_{1} & 0 \\
0 & i \Gamma_{2} Q_{2}
\end{array}\right)_{\sigma}\right] .
$$

Using the same logic that produced (B22) with $\hat{H}=\left(\frac{\alpha_{1} \sigma_{z}+\alpha_{2} \mathbf{1}_{\sigma}}{2}\right)=i \Gamma_{1} P_{1}+i \Gamma_{2} P_{2}$ for $P_{1}=\frac{1+\sigma_{z}}{2}$ and $P_{2}=\frac{1-\sigma_{z}}{2}$, and including a test function $f$, we calculate

$$
\begin{aligned}
& \left(g_{+}^{-1}-i \hat{H} \tau^{3}+i \Gamma_{1} Q_{1} P_{1}+i \Gamma_{2} Q_{2} P_{2}\right)(\hat{H})^{-1}\left(g_{-}^{-1}+i \hat{H} \tau^{3}-i \Gamma_{1} Q_{1} P_{1}-i Q_{2} P_{2}\right) f \\
& \quad=g_{+}^{-1} \hat{H}^{-1} g_{-}^{-1} f+\not\left(Q_{V} \mathbf{1}_{\sigma}+Q_{A} \sigma_{z}\right) f+(\not \nabla f)\left(Q_{V} \mathbf{1}_{\sigma}+Q_{A} \sigma_{z}\right)-\left(Q_{V} \mathbf{1}_{\sigma}+Q_{A} \sigma_{z}\right)(\not \nabla f) \\
& \quad=g_{+}^{-1} \hat{H}^{-1} g_{-}^{-1} f+\not\left(Q_{V} \mathbf{1}_{\sigma}+Q_{A} \sigma_{z}\right) f-2 Q_{A} \sigma_{z}(\not \nabla f),
\end{aligned}
$$

where we have used $Q_{1}^{2}=Q_{2}^{2}=\mathbf{1}$ and introduced the "vector" $Q_{V}=\frac{1}{2}\left(Q_{1}+Q_{2}\right)$ and "axial" $Q_{A}=\frac{1}{2}\left(Q_{1}-Q_{2}\right)$ matrix bosons. The crucial term that leads to a mass for $Q_{A}$ is $-2 Q_{A} \sigma_{z} \not$. We find

$$
\begin{aligned}
& \operatorname{Re}\left[S\left[Q_{1}, Q_{2}\right]\right]=\frac{1}{2} \operatorname{Tr} \ln \left[\left(g_{+}^{-1} \hat{H}^{-1} g_{-}^{-1}\right)\left(\mathbf{1}+\left(g_{-} \hat{H} g_{+}\right)\left(\not \nabla Q_{V}+\not \nabla Q_{A} \sigma_{z}-2 Q_{A} \sigma_{z} \not \nabla\right)\right)\right] \\
& \left.=\frac{1}{16} \operatorname{Tr}\left[\left(g_{R}-g_{A}\right)\left(\not \nabla Q_{V}+\not \triangleright Q_{A} \sigma_{z}-2 Q_{A} \sigma_{z} \not\right)\right)\left(g_{R}-g_{A}\right)\left(\not \nabla Q_{V}+\not \nabla Q_{A} \sigma_{z}-2 Q_{A} \sigma_{z} \not\right)\right] \text {. }
\end{aligned}
$$

Consider the last term, quadratic in $Q_{A} \not \mathbf{D}:$

$$
\begin{aligned}
& \frac{1}{4} \operatorname{Tr}\left[\left(g_{R}-g_{A}\right)\left(Q_{A} \sigma_{z} \not\right)\left(g_{R}-g_{A}\right)\left(Q_{A} \sigma_{z} \not\right)\right] \\
& \quad=\int_{x, x_{1}, x_{2}, x_{3}} \int_{k_{1} k_{2} k_{3} k_{4}} \frac{1}{4} \operatorname{Tr}\left[\left\langle x\left|\left(g_{R}-g_{A}\right)\right| k_{1}\right\rangle\left\langle k_{1} \mid x_{1}\right\rangle\left\langle x_{1}\left|Q_{A} \sigma_{z} \not\right| k_{2}\right\rangle\left\langle k_{2} \mid x_{2}\right\rangle\left\langle x_{2}\left|\left(g_{R}-g_{A}\right)\right| k_{3}\right\rangle\left\langle k_{3} \mid x_{3}\right\rangle\left\langle x_{3}\left|Q_{A} \sigma_{z} \not\right| k_{4}\right\rangle\left\langle k_{4} \mid x\right\rangle\right] \\
& \quad=\frac{-1}{4} \int_{k_{1}, k_{2}} \operatorname{Tr}\left[\left.\left.\left(g_{R}-g_{A}\right)\right|_{k_{1}} Q_{A}\left(k_{2}-k_{1}\right) \sigma_{z} k_{2}\left(g_{R}-g_{A}\right)\right|_{k_{2}} Q_{A}\left(k_{1}-k_{2}\right) \sigma_{z} \psi_{1}\right] \\
& \quad=\frac{-1}{4} \int_{p} Q_{A}(p) Q_{A}(-p) \int_{k_{1}} \operatorname{Tr}\left[\left.\left.\left(g_{R}-g_{A}\right)\right|_{k_{1}} \sigma_{z}\left(\not p+\not \psi_{1}\right)\left(g_{R}-g_{A}\right)\right|_{k_{1}+p} \sigma_{z} \not \psi_{1}\right]
\end{aligned}
$$




$$
\begin{aligned}
& \equiv \frac{-1}{4} \int_{p} Q_{A}(p) Q_{A}(-p) F(p) \\
& =\frac{-1}{4} \int_{p} Q_{A}(p) Q_{A}(-p)\left(F(p=0)+\left.\frac{\partial F}{\partial \not p}\right|_{p=0} \not p+\left.\frac{1}{2} \frac{\partial^{2} F}{\partial \not p^{2}}\right|_{p=0} \not p^{2}+\cdots\right),
\end{aligned}
$$

where we made the change of variables $k_{2}-k_{1} \equiv p$. Next, examine the two crossing terms containing $Q_{A} \not$ and $\not \nabla Q_{A}$ :

$$
\begin{aligned}
& \frac{1}{16} \operatorname{Tr}\left[\left(g_{R}-g_{A}\right)\left(\not \nabla Q_{A} \sigma_{z}\right)\left(g_{R}-g_{A}\right)\left(-2 Q_{A} \sigma_{z} \not \mathbf{W}\right)+\left(g_{R}-g_{A}\right)\left(-2 Q_{A} \sigma_{z} \not \mathbf{W}\right)\left(g_{R}-g_{A}\right)\left(\not \nabla Q_{A} \sigma_{z}\right)\right] \\
& \quad=\frac{-1}{4} \operatorname{Tr}\left[\left(g_{R}-g_{A}\right)\left(\not \nabla Q_{A} \sigma_{z}\right)\left(g_{R}-g_{A}\right)\left(Q_{A} \sigma_{z} \not\right)\right] \\
& \quad=\frac{-1}{4} \int_{d p} Q_{A}(p) Q_{A}(-p) \int_{d k_{1}} \operatorname{Tr}\left[\left.\left.\left(g_{R}-g_{A}\right)\right|_{k_{1}} \sigma_{z} k_{1}\left(g_{R}-g_{A}\right)\right|_{p+k_{1}} \sigma_{z} k_{1}\right] .
\end{aligned}
$$

Combining Eqs. (B16) and (B18), the total mass term for $Q_{A}$ is $\frac{-1}{2} \int_{d p_{1}} Q_{A}\left(p_{1}\right) Q_{A}\left(-p_{1}\right) F(0)$. The function $F(0) \neq 0$ generally: $Q_{A}$ is only massless if it is tuned to criticality. This is in sharp contrast to $Q_{V}$, which is massless because it is a Goldstone boson. Thus, $Q_{A}$ is generally massive and we neglect it at low energies.

\section{Real part of $S_{\mathrm{NLSM}}$}

To compute the real part of $S_{\mathrm{NLSM}}$ we directly compute the fermion determinant implied by (B4):

$$
\operatorname{Re}\left(S_{\mathrm{NLSM}}\right)+i \operatorname{Im}\left(S_{\mathrm{NLSM}}\right)=\operatorname{Tr} \ln \left(\left(M_{1} \sigma_{0}-M_{2} \sigma_{z}+i \sigma_{x} \partial_{x}-i \sigma_{y} \partial_{y}\right)+i \frac{\alpha_{1} \sigma_{z}+\alpha_{2} \sigma_{0}}{2} Q\right) .
$$

For any operator $\hat{X}$ with determinant $\operatorname{det} \hat{X}=R e^{i \theta}$, we seek $\ln R$. Let $i \hat{C} \equiv i\left(\frac{\alpha_{1} \sigma_{z}+\alpha_{2} \sigma_{0}}{2}\right) Q$ and $\hat{B} \equiv M_{1} \sigma_{0}-M_{2} \sigma_{z}+i \sigma \partial_{x}-$ $i \sigma_{y} \partial_{y}$, then we can decompose the operator in $2 \times 2 \sigma$ space using the identities

$$
\begin{gathered}
\operatorname{det}[i \hat{C}+\hat{B}]=\operatorname{det}[i \hat{C}]+\operatorname{det}[\hat{B}]+i \operatorname{det}[\hat{C}] \operatorname{Tr}\left[\hat{C}^{-1} \hat{B}\right], \\
\operatorname{det}\left[(\hat{B}+i \hat{C}) \hat{H}^{-1}(\hat{B}-i \hat{C}) \hat{H}\right]=|\operatorname{det}[i \hat{C}+\hat{B}]|^{2},
\end{gathered}
$$

where $\hat{H}$ is any constant matrix in $\sigma$ space that satisfies $[\hat{C}, \hat{H}]=0$. We choose $\hat{H}=\left(\frac{\alpha_{1} \sigma_{z}+\alpha_{2} \sigma_{0}}{2}\right)$. Up to an unimportant constant that we drop, we find

$$
\begin{aligned}
\operatorname{Re}\left(S_{\mathrm{NLSM}}\right) & =\frac{1}{2} \operatorname{Tr} \ln \left[\left(g_{+}^{-1}-i \hat{H} \tau^{3}+i \hat{H} Q\right)(\hat{H})^{-1}\left(g_{-}^{-1}+i \hat{H} \tau^{3}-i \hat{H} Q\right)\right] \\
& =\frac{1}{2} \operatorname{Tr}\left[\left(\frac{i}{2}\left(g_{R}-g_{A}\right) \not \nabla Q\right)-\frac{1}{2}\left(\frac{i}{2}\left(g_{R}-g_{A}\right) \not \nabla Q\right)\left(\frac{i}{2}\left(g_{R}-g_{A}\right) \not \nabla Q\right)\right] \\
& =\frac{1}{16} \operatorname{Tr}_{\sigma}\left[\left(g_{R}-g_{A}\right) \kappa_{a}\left(g_{R}-g_{A}\right) \kappa_{b}\right] \operatorname{Tr}\left[\nabla_{a} Q \nabla_{b} Q\right] \\
& \equiv \frac{-S_{j k}}{8} \operatorname{Tr}\left[\nabla_{j} Q \nabla_{k} Q\right],
\end{aligned}
$$

where $\frac{\not}{i} \equiv \kappa_{x} \frac{\partial_{x}}{i}+\kappa_{y} \frac{\partial_{y}}{i}, \kappa_{x}=\sigma_{x}, \kappa_{y}=-\sigma_{y}$. We identify $S_{j k}=\sigma_{x x}^{\text {cf }} \delta_{j k}$ as the DC longitudinal conductivity:

$$
\begin{aligned}
\sigma_{x x}^{\mathrm{cf}} & =-\frac{1}{2} \operatorname{Tr}\left[\left(g_{R}-g_{A}\right) \sigma_{x}\left(g_{R}-g_{A}\right) \sigma_{x}\right] \\
& =1+\frac{\left(4 m_{1}^{2}-4 m_{2}^{2}-\alpha_{1}^{2}+\alpha_{2}^{2}\right)\left(\pi+2 \operatorname{arccot}\left[\frac{\left|\mathrm{m}_{1} \alpha_{2}-\mathrm{m}_{2} \alpha_{1}\right|}{4 \mathrm{~m}_{1}^{2}-4 \mathrm{~m}_{2}^{2}+\alpha_{1}^{2}-\alpha_{2}^{2}}\right]\right)}{8\left|m_{1} \alpha_{2}+m_{2} \alpha_{1}\right|} \\
& \approx \frac{\pi\left(m_{1}^{2}-m_{2}^{2}\right)}{\left|m_{1} \alpha_{2}-m_{2} \alpha_{1}\right|} \gg 1 .
\end{aligned}
$$

In these expressions we have used $m_{1}=\frac{1}{2 n} \operatorname{Tr} M_{1}$ and $m_{2}=-\frac{1}{2 n} \operatorname{Tr} M_{2}$. The last expression uses the weak disorder limit, i.e., $O\left(E_{F}\right) \approx O\left(\left|m_{1}\right|\right) \gg \alpha_{2}, \alpha_{1}$. 
Here and below we use the DC $(\omega \rightarrow 0)$ limit of the Kubo formula:

$$
\begin{aligned}
\sigma_{i j}^{\mathrm{cf}}(q \rightarrow 0, \omega+i \eta)=- & \int \frac{d^{2} \boldsymbol{r}^{\prime}}{V} \int d^{2} \boldsymbol{r} \frac{1}{2 \pi \omega} \int_{-\infty}^{\infty} d z \\
& \times\left([f(z)-f(z-\omega)] \operatorname{Tr}\left[\kappa_{i} G_{z}^{R}\left(r, r^{\prime}\right) \kappa_{j} G_{z-\omega}^{A}\left(r^{\prime}, r\right)\right]+f(z) \operatorname{Tr}\left[\kappa_{i} G_{z+\omega}^{R}\left(r, r^{\prime}\right) \kappa_{j} G_{z}^{R}\left(r^{\prime}, r\right)\right]\right. \\
& \left.-f(z) \operatorname{Tr}\left[\kappa_{i} G_{z}^{A}\left(r, r^{\prime}\right) \kappa_{j} G_{z-\omega}^{A}\left(r^{\prime}, r\right)\right]\right) .
\end{aligned}
$$

For the Dirac theory, the SCBA Green's function is

$$
G_{D}\left(\epsilon+E_{F} ; \omega\right)_{R / A}=\frac{1}{\left(\epsilon+E_{F}+\omega\right) \sigma_{0}-m_{D} \sigma_{z}+i \partial_{x} \sigma_{x}-i \partial_{y} \sigma_{y} \pm i \frac{\alpha_{1} \sigma_{z}+\alpha_{2} \sigma_{0}}{2}} .
$$

For the linearized HLR theory,

$$
g\left(\epsilon+E_{F} ; \omega\right)_{R / A}=\frac{1}{\left(\epsilon+E_{F}\right) \sigma_{0}+\omega P_{1}+2 m P_{2}+i \partial_{x} \sigma_{x}-i \partial_{y} \sigma_{y} \pm i \frac{\alpha_{1} \sigma_{z}+\alpha_{2} \sigma_{0}}{2}},
$$

where $P_{1}=\frac{1+\sigma_{z}}{2}$ and $P_{2}=\frac{1-\sigma_{z}}{2}$ and we have inserted the specific masses for the HLR (3.6) and Dirac (3.7) theories using (3.8). The frequency term should be thought of as a "mass" term in the Hamiltonian instead of the physical frequency appearing in (B25). Notice that since we have included the Fermi level in the above Greens function, we only need to perform the energy integral $\int d z f(z)$ up to zero. We comment that a direct calculation of the Hall conductivity using the above SCBA Green's functions agrees with the results below, up to the crucial additive term equal to $\frac{e^{2}}{2 h}$.

\section{Imaginary part of $S_{\mathrm{NLSM}}$}

To calculate the imaginary part of $S_{\mathrm{NLSM}}$ in (B4), we first set $n=1$ and perform the gauge transformation $\Psi(\mathbf{x}) \rightarrow U(\mathbf{x}) \Psi(\mathbf{x})$. This introduces the gauge field $A_{j}(\mathbf{x})=i U^{\dagger}(\mathbf{x}) \partial_{j} U(\mathbf{x})=\sum_{b=1}^{3} A_{j}^{b} \tau_{b}$, where $\left(\tau_{1}, \tau_{2}, \tau_{3}\right)$ are the Pauli matrices in retardedadvanced space. We determine $\operatorname{Im}\left(S_{\mathrm{NLSM}}\right)$ from the imaginary part of the expectation value of the current (calculated to linear order in $A_{j}$ ):

$$
i \frac{\delta\left(\operatorname{Im} S_{\mathrm{NLSM}}\right)}{\delta A_{j}^{b}}=\frac{\delta S_{\mathrm{top}}^{\mathrm{I}}}{\delta A_{j}^{b}}+\frac{\delta S_{\mathrm{top}}^{\mathrm{II}}}{\delta A_{j}^{b}},
$$

where $S_{\text {top }}^{\mathrm{I}}$ and $S_{\text {top }}^{\mathrm{II}}$ are the linear (quantum) and quadratic (classical) in $A_{j}$ contributions to the imaginary part of $S_{\mathrm{NLSM}}$ [75].

$$
\text { a. } S_{\text {top }}^{I I}
$$

We begin with the classical contribution $S_{\text {top }}^{\mathrm{II}}$. By direct evaluation we find

$$
\begin{aligned}
S_{\text {top }}^{\mathrm{II}} & =\frac{1}{2} \operatorname{Tr}\left[\kappa_{\mu} A_{\mu} g_{+} \kappa_{\nu} A_{\nu} g_{+}-\kappa_{\mu} A_{\mu} g_{-} \kappa_{\nu} A_{\nu} g_{-}\right] \\
& =\frac{1}{2} \operatorname{Tr}\left[\kappa_{\mu} A_{\mu}\left(g_{+}+g_{-}\right) \kappa_{\nu} A_{\nu}\left(g_{+}-g_{-}\right)\right] \\
& =\frac{i}{2} \operatorname{Tr}_{\sigma}\left[\kappa_{\mu}\left(g_{R}+g_{A}\right) \kappa_{\nu}\left(g_{R}-g_{A}\right)\right] \operatorname{Tr}_{\tau}\left[A_{\mu} A_{\nu} \tau_{3}\right] \\
& =\frac{i}{4} \operatorname{Tr}_{\sigma}\left[\kappa_{x}\left(g_{R}+g_{A}\right) \kappa_{y}\left(g_{R}-g_{A}\right)\right] \operatorname{Tr}_{\tau}\left[Q\left(\partial_{x} Q\right)\left(\partial_{y} Q\right)\right] \\
& \equiv i \frac{\theta^{\mathrm{II}}}{2 \pi} \operatorname{Tr}_{\tau}\left[Q\left(\partial_{x} Q\right)\left(\partial_{y} Q\right)\right],
\end{aligned}
$$

where we used $\operatorname{Tr}_{\tau}\left[Q\left(\partial_{x} Q\right)\left(\partial_{y} Q\right)\right]=4 i\left(A_{i}^{1} A_{j}^{2}-A_{i}^{2} A_{j}^{1}\right) \epsilon_{i j} \cdot \frac{\theta^{\mathrm{II}}}{2 \pi}$ is the classical contribution $\sigma_{x y}^{\mathrm{I}}$ to the DC Hall conductivity $\sigma_{x y}^{\text {cf }}=$ $\sigma_{x y}^{\mathrm{I}}+\sigma_{x y}^{\mathrm{II}}$ :

$$
\sigma_{x y}^{\mathrm{I}}=\frac{1}{2 \pi}\left(\frac{m_{2} \Gamma_{+}+m_{1} \Gamma_{-}}{m_{1} \Gamma_{+}+m_{2} \Gamma_{-}}\right)\left(\arctan \left[\frac{2\left(\Gamma_{+}+\Gamma_{-}\right)}{2\left(m_{1}-m_{2}\right)}\right]+\arctan \left[\frac{2\left(\Gamma_{+}-\Gamma_{-}\right)}{2\left(m_{1}+m_{2}\right)}\right]\right) .
$$

The quantum contribution $\sigma_{x y}^{\mathrm{II}}$ to the Hall conductivity equals $\frac{\theta^{\mathrm{I}}}{2 \pi}$ (modulo 1); it is calculated in the next section. 


$$
\text { b. } S_{\text {top }}^{I}
$$

$S_{\text {top }}^{\mathrm{I}}$ obtains from a result first obtained by Goldstone and Wilczek [76]; below we follow the treatment in [83]. We first introduce the "mass field"

$$
\Phi_{m}(0) \equiv M_{1} \sigma_{0}-M_{2} \sigma_{z}+i\left(\frac{\alpha_{1} \sigma_{z}+\alpha_{2} \sigma_{0}}{2}\right) \tau_{3} .
$$

For this calculation we will treat $\Phi_{m}(0)$ as a spatially varying field $\Phi_{m}(\mathbf{x})$ that takes its fixed point value (B31) at the end of the calculation. We write the mass field as

$$
\Phi_{m}(x) \equiv\left[m_{1 a}(x) \tau_{3}+m_{1 b}(x) \tau_{0}\right] \sigma_{0}+\left[m_{2 a}(x) \tau_{3}-m_{2 b}(x) \tau_{0}\right] \sigma_{z} .
$$

Notice that $m_{1}=m_{1 a}(0)$ and $m_{2}=m_{2 a}(0)$. The real space Green's function is then expanded about uniform $\Phi_{m}$ as

$$
S\left(\Phi_{m}\right)=\int \frac{d p_{1} d p_{2}}{(2 \pi)^{2}} \frac{1}{i \kappa_{\mu} D_{\mu}+\Phi_{m}(x)} e^{i \boldsymbol{p} \cdot(x-y)}=S_{0}+\left\{-S_{0}\left[x_{\nu} \partial_{\nu} \Phi_{m}(0)\right] S_{0}\right\}+\cdots,
$$

where we drop the "..." in what follows. $S_{0}$ is the Green's function for uniform $\Phi_{m}$ with $\left.S_{0}\right|_{A_{j}=0}=g_{+}$, given in (B6).

The quantum current $\frac{\delta S_{\text {top }}^{\mathrm{I}}}{\delta A_{j}^{b}}$ is then computed as

$$
\frac{\delta S_{\text {top }}^{\mathrm{I}}}{\delta A_{j}^{b}}=-\lim _{y \rightarrow x} \operatorname{Tr}\left[\left\langle\kappa_{j} \tau_{b} S(x, y)\right\rangle\right] \approx\left\langle x\left|\operatorname{Tr}\left[\kappa_{j} \tau_{b} S_{0} \partial_{k} \Phi_{m}(0) i S_{0} \kappa_{k} S_{0}\right]\right| x\right\rangle,
$$

where we use $\left\langle x^{\prime}\left|S_{0} \hat{r}_{k}\right| x\right\rangle=\left\langle x^{\prime}\left|i S_{0} \kappa_{k} S_{0}\right| x\right\rangle$. We set the gauge potential in the Green's function $S_{0}$ to zero to find

$$
\begin{aligned}
\frac{\delta S_{\text {top }}^{\mathrm{I}}}{\delta A_{j}^{b}}= & \int \frac{d q_{x} d q_{y}}{(2 \pi)^{2}} \operatorname{Tr}\left[\kappa_{j} \tau_{b} \frac{1}{q+\Phi_{m}(0)} \partial_{k} \Phi_{m}(0) \frac{1}{q+\Phi_{m}(0)} i \kappa_{k} \frac{1}{q+\Phi_{m}(0)}\right] \\
= & \frac{1}{2 \pi} \epsilon_{j k} \delta_{b 3}\left[\frac{\left(m_{1 a}+m_{1 b}\right) \partial_{k}\left(-m_{2 a}+m_{2 b}\right)-\left(-m_{2 a}+m_{2 b}\right) \partial_{k}\left(m_{1 a}+m_{1 b}\right)}{\left(-m_{2 a}+m_{2 b}\right)^{2}-\left(m_{1 a}+m_{1 b}\right)^{2}}\right] \\
& \left.\quad-\frac{\left(-m_{1 a}+m_{1 b}\right) \partial_{k}\left(m_{2 a}+m_{2 b}\right)-\left(m_{2 a}+m_{2 b}\right) \partial_{k}\left(-m_{1 a}+m_{1 b}\right)}{\left(m_{2 a}+m_{2 b}\right)^{2}-\left(-m_{1 a}+m_{1 b}\right)^{2}}\right] \\
\equiv & \frac{i}{2 \pi} \delta_{b 3} \epsilon_{j k} \partial_{k} \theta^{\mathrm{I}},
\end{aligned}
$$

where $q \equiv \kappa_{x} p_{x}+\kappa_{y} p_{y}$. We now deduce $S_{\text {top }}^{\mathrm{I}}$ by coupling this current to $A_{j}^{b}$ and then performing an integration by parts:

$$
S_{\text {top }}^{\mathrm{I}}=\int d^{2} x \sum_{b=1}^{3} A_{j}^{b} \frac{\delta S_{\text {top }}^{\mathrm{I}}}{\delta A_{j}^{b}}=\frac{i}{2 \pi} \int d^{2} x A_{j}^{3} \epsilon_{j k} \partial_{k} \theta^{\mathrm{I}}=-\frac{1}{4 \pi} \int d^{2} x \theta^{\mathrm{I}} \epsilon_{j k} \operatorname{Tr}_{\tau}\left[\tau^{3} \partial_{k} A_{j}\right] .
$$

It remains to determine $\theta^{\mathrm{I}}$ for the specific fixed point value $\Phi_{m}(0)(\mathrm{B} 31)$. The detailed discussion below complements the presentation of this in the main text. We replace $\alpha_{1} / 2=\Gamma_{-}$and $\alpha_{2} / 2=\Gamma_{+}$, and introduce the superscript $s=R, A$ to label the diagonal retarded and advanced components in $\tau$ space of

$$
\varphi_{1}=m_{1} \tau^{0}+i \Gamma_{+} \tau^{3}, \quad \varphi_{2}=-m_{2} \tau^{0}+i \Gamma_{-} \tau^{3} .
$$

Thus, we may also specify the diagonal components of $\Phi_{m}$ with this superscript,

$$
\Phi_{m}^{s}(x)=-\varphi_{2}^{s}(x) \sigma_{z}+\varphi_{1}^{s}(x) \sigma_{0} \equiv\left(\begin{array}{cc}
-e^{-i \chi_{2}^{s}} & 0 \\
0 & e^{i \chi_{1}^{s}}
\end{array}\right) .
$$

Equation (B35) indicates that the retarded and advanced contributions to $\theta^{\mathrm{I}}$ are fully separable, i.e., $\theta^{\mathrm{I}}$ only couples to $\frac{1}{2} \operatorname{Tr}\left(\tau^{3} A_{j}\right)=A_{j}^{3}$. This allows us to determine $\theta^{\mathrm{I}}$ as the contribution from the $U(1)$ subgroup of $\mathrm{SU}(2)$ associated with $\tau^{3}$. Plugging (B38) into (B35), we find

$$
-\partial_{k} \theta^{\mathrm{I}}=\partial_{k} \frac{\chi_{1}^{R}+\chi_{2}^{R}-\chi_{1}^{A}-\chi_{2}^{A}}{2}
$$

Note that $\chi_{1}^{s}$ and $\chi_{2}^{s}$ are generally complex. We restrict $\operatorname{Re}\left[\chi_{1,2}\right] \in[0, \pi)$. We now specialize to the masses associated with the HLR theory by setting $m_{1}=E_{F} / 2+m$ and $m_{2}=E_{F} / 2-m$; similar manipulations yield $\theta^{\mathrm{I}}$ for the Dirac theory. We use $\varphi_{1}+\varphi_{2}=e^{i \chi_{1}}, \varphi_{1}-\varphi_{2}=-e^{-i \chi_{2}}$, and (B31) to find the equations:

$$
\begin{array}{ll}
2 m+i\left(\Gamma_{+}+\Gamma_{-}\right)=e^{i \chi_{1}^{R}}, & E_{F}+i\left(\Gamma_{+}-\Gamma_{-}\right)=-e^{-i \chi_{2}^{R},} \\
2 m-i\left(\Gamma_{+}+\Gamma_{-}\right)=e^{i \chi_{1}^{A}}, & E_{F}-i\left(\Gamma_{+}-\Gamma_{-}\right)=-e^{-i \chi_{2}^{A}} .
\end{array}
$$


For the retarded component $\chi_{1,2}^{R}$ we find

$$
\begin{gathered}
\operatorname{Re}\left[\chi_{1}^{R}\right]=\arctan \left[\frac{\Gamma_{+}+\Gamma_{-}}{2 m}\right], \quad \operatorname{Im}\left[\chi_{1}^{R}\right]=\frac{-1}{2} \log \left[(2 m)^{2}+\left(\Gamma_{+}+\Gamma_{-}\right)^{2}\right], \\
\operatorname{Re}\left[\chi_{2}^{R}\right]=\pi-\arctan \left[\frac{\Gamma_{+}-\Gamma_{-}}{E_{F}}\right], \quad \operatorname{Im}\left[\chi_{2}^{R}\right]=\frac{-1}{2} \log \left[\left(E_{F}\right)^{2}+\left(\Gamma_{+}-\Gamma_{-}\right)^{2}\right] .
\end{gathered}
$$

Due to the restriction $\operatorname{Re}\left[\chi_{1,2}^{s}\right] \in[0, \pi)$, we need to perform a charge conjugation on the advanced fermions using the definition in (3.56). It is here that we use the fact that $\theta^{\mathrm{I}}$ is only sensitive to the Abelian component $\frac{1}{2} \operatorname{Tr}\left(\tau^{3} A_{j}\right)$ of $A_{j}$. Charge conjugation flips the sign of mass term involving $\sigma_{0}$ as well as the gauge coupling of the advanced fermion to $A_{j}^{3}$ : The part of the action including the advanced fermion only transforms as

$$
\begin{gathered}
S_{A}=\Psi_{A}^{\dagger}\left(m_{1} \sigma_{0}+m_{2} \sigma_{z}+i \sigma_{x}\left(\partial_{x}-i A_{x}^{3}\right)-i \sigma_{y}\left(\partial_{y}-i A_{y}^{3}\right)-i \frac{\alpha_{1} \sigma_{z}+\alpha_{2} \sigma_{0}}{2}\right) \Psi_{A}, \\
\mathcal{C} S_{A} \mathcal{C}=\Psi_{A}^{\dagger}\left(-m_{1} \sigma_{0}+m_{2} \sigma_{z}+i \sigma_{x}\left(\partial_{x}+i A_{x}^{3}\right)-i \sigma_{y}\left(\partial_{y}+i A_{y}^{3}\right)-i \frac{\alpha_{1} \sigma_{z}-\alpha_{2} \sigma_{0}}{2}\right) \Psi_{A} .
\end{gathered}
$$

After performing this charge conjugation, we find the current $\frac{\delta S_{\text {top }}^{\mathrm{I}}}{\delta A_{j}^{b}}$ equals

$$
\begin{aligned}
& \frac{\delta S_{\text {top }}^{\mathrm{I}}}{\delta A_{j}^{b}}=\frac{1}{2 \pi} \epsilon_{j k} \delta_{b 3}[ {\left[\frac{\left(m_{1 a}+m_{1 b}\right) \partial_{k}\left(-m_{2 a}+m_{2 b}\right)-\left(-m_{2 a}+m_{2 b}\right) \partial_{k}\left(m_{1 a}+m_{1 b}\right)}{\left(-m_{2 a}+m_{2 b}\right)^{2}-\left(m_{1 a}+m_{1 b}\right)^{2}}\right.} \\
&\left.+\frac{(-1)\left(-m_{1 a}+m_{1 b}\right) \partial_{k}\left(m_{2 a}+m_{2 b}\right)-\left(m_{2 a}+m_{2 b}\right)(-1) \partial_{k}\left(-m_{1 a}+m_{1 b}\right)}{\left(m_{2 a}+m_{2 b}\right)^{2}-\left(-m_{1 a}+m_{1 b}\right)^{2}}\right] \\
& \equiv \frac{i}{2 \pi} \delta_{b 3} \epsilon_{j k} \partial_{k} \theta^{\mathrm{I}} .
\end{aligned}
$$

The retarded contribution to $\theta^{\mathrm{I}}$ is unchanged since only the advanced fermions were charge conjugated. The advanced fermion mass becomes

$$
\Phi_{m}^{A}(x)=-\varphi_{2}^{A}(x) \sigma_{z}+(-1) \varphi_{1}^{A}(x) \sigma_{0} \equiv\left(\begin{array}{cc}
-e^{-i \tilde{\chi}_{2}^{A}} & 0 \\
0 & e^{i \tilde{\chi}_{1}^{A}}
\end{array}\right) .
$$

This results in the equations for $\tilde{\chi}_{1,2}^{A}$ :

$$
\begin{array}{cc}
2 m-i\left(\Gamma_{+}+\Gamma_{-}\right)=e^{i \tilde{\chi}_{2}^{A}}, & -E_{F}+i\left(\Gamma_{+}-\Gamma_{-}\right)=e^{i \tilde{\chi}_{1}^{A}}, \\
\operatorname{Re}\left[\tilde{\chi}_{2}^{A}\right]=\arctan \left[\frac{\Gamma_{+}+\Gamma_{-}}{2 m}\right], & \operatorname{Re}\left[\tilde{\chi}_{1}^{A}\right]=\pi-\arctan \left[\frac{\Gamma_{+}-\Gamma_{-}}{E_{F}}\right] .
\end{array}
$$

Since charge conjugation also flips relative sign between retarded and advanced contributions in Eq. (B39), we have

$$
\begin{aligned}
\theta^{\mathrm{I}} & =-\frac{1}{2}\left[\left(\operatorname{Re}\left[\chi_{1}^{R}\right]+\operatorname{Re}\left[\chi_{2}^{R}\right]+\operatorname{Re}\left[\tilde{\chi}_{1}^{A}\right]+\operatorname{Re}\left[\tilde{\chi}_{2}^{A}\right]\right)\right] \\
& =\pi+\arctan \left[\frac{\Gamma_{+}-\Gamma_{-}}{m_{1}+m_{2}}\right]-\arctan \left[\frac{\Gamma_{+}+\Gamma_{-}}{m_{1}-m_{2}}\right] .
\end{aligned}
$$

We have used $\theta^{\mathrm{I}}=0(\bmod 2 \pi)$ to fix the coefficient of $\pi$ to be unity and replaced the HLR-specific masses by the general masses $m_{1}$ and $m_{2}$.

[1] The Quantum Hall Effect, edited by R. Prange and S. Girvin, Graduate Texts in Contemporary Physics (Springer, Berlin, 1987).

[2] Perspectives in Quantum Hall Effects: Novel Quantum Liquids in Low-Dimensional Semiconductor Structures, edited by S. Das Sarma and A. Pinczuk (Wiley-VCH, Berlin, 1996).

[3] R. B. Laughlin, Quantized Hall conductivity in two dimensions, Phys. Rev. B 23, 5632 (1981).

[4] H. Aoki and T. Ando, Effect of localization on the Hall conductivity in the two-dimensional system in strong magnetic fields, Solid State Commun. 38, 1079 (1981).
[5] B. Huckestein, Scaling theory of the integer quantum Hall effect, Rev. Mod. Phys. 67, 357 (1995).

[6] S. L. Sondhi, S. M. Girvin, J. P. Carini, and D. Shahar, Continuous quantum phase transitions, Rev. Mod. Phys. 69, 315 (1997).

[7] Z. Wang, M. P. A. Fisher, S. M. Girvin, and J. T. Chalker, Short-range interactions and scaling near integer quantum Hall transitions, Phys. Rev. B 61, 8326 (2000).

[8] K. Slevin and T. Ohtsuki, Critical exponent for the quantum Hall transition, Phys. Rev. B 80, 041304(R) (2009).

[9] H. Obuse, A. R. Subramaniam, A. Furusaki, I. A. Gruzberg, and A. W. W. Ludwig, Conformal invariance, multifractality, 
and finite-size scaling at anderson localization transitions in two dimensions, Phys. Rev. B 82, 035309 (2010).

[10] M. Amado, A. V. Malyshev, A. Sedrakyan, and F. DomínguezAdame, Numerical Study of the Localization Length Critical Index in a Network Model of Plateau-Plateau Transitions in the Quantum Hall Effect, Phys. Rev. Lett. 107, 066402 (2011).

[11] J. P. Dahlhaus, J. M. Edge, J. Tworzydło, and C. W. J. Beenakker, Quantum Hall effect in a one-dimensional dynamical system, Phys. Rev. B 84, 115133 (2011).

[12] I. C. Fulga, F. Hassler, A. R. Akhmerov, and C. W. J. Beenakker, Topological quantum number and critical exponent from conductance fluctuations at the quantum Hall plateau transition, Phys. Rev. B 84, 245447 (2011).

[13] K. Slevin and T. Ohtsuki, Finite size scaling of the ChalkerCoddington model, Int. J. Mod. Phys. 11, 60 (2012).

[14] H. Obuse, I. A. Gruzberg, and F. Evers, Finite-Size Effects and Irrelevant Corrections to Scaling Near the Integer Quantum Hall Transition, Phys. Rev. Lett. 109, 206804 (2012).

[15] Q. Zhu, P. Wu, R. N. Bhatt, and X. Wan, Localization-length exponent in two models of quantum Hall plateau transitions, Phys. Rev. B 99, 024205 (2019).

[16] M. Puschmann, P. Cain, M. Schreiber, and T. Vojta, Integer quantum Hall transition on a tight-binding lattice, Phys. Rev. B 99, 121301(R) (2019).

[17] W. Li, C. L. Vicente, J. S. Xia, W. Pan, D. C. Tsui, L. N. Pfeiffer, and K. W. West, Scaling in Plateau-to-Plateau Transition: A Direct Connection of Quantum Hall Systems with the Anderson Localization Model, Phys. Rev. Lett. 102, 216801 (2009).

[18] W. Li, J. S. Xia, C. Vicente, N. S. Sullivan, W. Pan, D. C. Tsui, L. N. Pfeiffer, and K. W. West, Crossover from the nonuniversal scaling regime to the universal scaling regime in quantum Hall plateau transitions, Phys. Rev. B 81, 033305 (2010).

[19] I. A. Gruzberg, A. Klümper, W. Nuding, and A. Sedrakyan, Geometrically disordered network models, quenched quantum gravity, and critical behavior at quantum Hall plateau transitions, Phys. Rev. B 95, 125414 (2017).

[20] R. Bondesan, D. Wieczorek, and M. Zirnbauer, Gaussian free fields at the integer quantum Hall plateau transition, Nucl. Phys. B 918, 52 (2017).

[21] B. Sbierski, E. J. Dresselhaus, J. E. Moore, and I. A. Gruzberg, Criticality of Two-Dimensional Disordered Dirac Fermions in the Unitary Class and Universality of the Integer Quantum Hall Transition, Phys. Rev. Lett. 126, 076801 (2021).

[22] M. R. Zirnbauer, The integer quantum Hall plateau transition is a current algebra after all, Nucl. Phys. B 941, 458 (2019).

[23] J. T. Chalker and G. J. Daniell, Scaling, Diffusion, and the Integer Quantized Hall Effect, Phys. Rev. Lett. 61, 593 (1988).

[24] M. P. A. Fisher, G. Grinstein, and S. M. Girvin, Presence of Quantum Diffusion in Two Dimensions: Universal Resistance at the Superconductor-Insulator Transition, Phys. Rev. Lett. 64, 587 (1990).

[25] D.-H. Lee and Z. Wang, Effects of Electron-Electron Interactions on the Integer Quantum Hall Transitions, Phys. Rev. Lett. 76, 4014 (1996).

[26] E. Fradkin, Field Theories of Condensed Matter Physics (Cambridge University Press, Cambridge, 2013).

[27] J. K. Jain, Composite Fermions (Cambridge University Press, Cambridge, 2007).

[28] J. K. Jain, Composite-Fermion Approach for the Fractional Quantum Hall Effect, Phys. Rev. Lett. 63, 199 (1989).
[29] S. C. Zhang, T. H. Hansson, and S. Kivelson, EffectiveField-Theory Model for the Fractional Quantum Hall Effect, Phys. Rev. Lett. 62, 82 (1989).

[30] A. Lopez and E. Fradkin, Fractional quantum Hall effect and Chern-Simons gauge theories, Phys. Rev B 44, 5246 (1991).

[31] B. I. Halperin, P. A. Lee, and N. Read, Theory of the half-filled Landau level, Phys. Rev. B 47, 7312 (1993).

[32] K. S. Huang, S. Raghu, and P. Kumar, Numerical Study of a Dual Representation of the Integer Quantum Hall Transition, Phys. Rev. Lett. 126, 056802 (2021).

[33] E. Shimshoni, S. L. Sondhi, and D. Shahar, Duality near quantum Hall transitions, Phys. Rev. B 55, 13730 (1997).

[34] A. Hui, E.-A. Kim, and M. Mulligan, Non-Abelian bosonization and modular transformation approach to superuniversality, Phys. Rev. B 99, 125135 (2019).

[35] P. Kumar, P. A. Nosov, and S. Raghu, Interaction effects on quantum Hall transitions: Dynamical scaling laws and superuniversality, arXiv:2006.11862.

[36] P. Kumar, S. Raghu, and M. Mulligan, Composite fermion Hall conductivity and the half-filled Landau level, Phys. Rev. B 99, 235114 (2019).

[37] P. Kumar, M. Mulligan, and S. Raghu, Topological phase transition underpinning particle-hole symmetry in the HalperinLee-Read theory, Phys. Rev. B 98, 115105 (2018).

[38] P. Kumar, M. Mulligan, and S. Raghu, Emergent reflection symmetry from nonrelativistic composite fermions, Phys. Rev. B 99, 205151 (2019).

[39] S. M. Girvin, Particle-hole symmetry in the anomalous quantum Hall effect, Phys. Rev. B 29, 6012 (1984).

[40] D. Shahar, D. C. Tsui, M. Shayegan, E. Shimshoni, and S. L. Sondhi, Evidence for charge-flux duality near the quantum Hall liquid-to-insulator transition, Science 274, 589 (1996).

[41] H. Goldman and E. Fradkin, Dirac composite fermions and emergent reflection symmetry about even-denominator filling fractions, Phys. Rev. B 98, 165137 (2018).

[42] J. Wang, Dirac Fermion Hierarchy of Composite Fermi Liquids, Phys. Rev. Lett. 122, 257203 (2019).

[43] D. Shahar, D. C. Tsui, M. Shayegan, R. N. Bhatt, and J. E. Cunningham, Universal Conductivity at the Quantum Hall Liquid to Insulator Transition, Phys. Rev. Lett. 74, 4511 (1995).

[44] W. Pan, W. Kang, M. P. Lilly, J. L. Reno, K. W. Baldwin, K. W. West, L. N. Pfeiffer, and D. C. Tsui, Particle-Hole Symmetry and the Fractional Quantum Hall Effect in the Lowest Landau Level, Phys. Rev. Lett. 124, 156801 (2020).

[45] S. A. Kivelson, D.-H. Lee, Y. Krotov, and J. Gan, Compositefermion Hall conductance at $v=1 / 2$, Phys. Rev. B 55, 15552 (1997).

[46] D. T. Son, Is the Composite Fermion a Dirac Particle? Phys. Rev. X 5, 031027 (2015).

[47] N. Seiberg, T. Senthil, C. Wang, and E. Witten, A duality web in $2+1$ dimensions and condensed matter physics, Ann. Phys. 374, 395 (2016).

[48] C. Wang, N. R. Cooper, B. I. Halperin, and A. Stern, ParticleHole Symmetry in the Fermion-Chern-Simons and Dirac Descriptions of a Half-Filled Landau Level, Phys. Rev. X 7, 031029 (2017).

[49] We have simplified the presentation in this paragraph by focusing on the HLR composite fermion theory [31]. In the Dirac composite fermion theory [46,47,51-53], particle-hole symmetry requires the Dirac composite fermions to exhibit vanishing 
Hall conductivity $\sigma_{x y}^{\text {cf }}=0$. Due to their nonzero Berry phase, localized free Dirac particles exhibit half-integer Hall conductivity.

[50] Note that noninteracting electrons in the presence of various types of quenched disorder can also exhibit particle-hole symmetric electrical transport [81].

[51] C. Wang and T. Senthil, Dual Dirac Liquid on the Surface of the Electron Topological Insulator, Phys. Rev. X 5, 041031 (2015).

[52] M. A. Metlitski and A. Vishwanath, Particle-vortex duality of two-dimensional Dirac fermion from electric-magnetic duality of three-dimensional topological insulators, Phys. Rev. B 93, 245151 (2016).

[53] A. Karch and D. Tong, Particle-Vortex Duality from 3D Bosonization, Phys. Rev. X 6, 031043 (2016).

[54] P. Kumar, Y. B. Kim, and S. Raghu, Self-duality of the integer quantum Hall to insulator transition: Composite fermion description, Phys. Rev. B 100, 235124 (2019).

[55] E. Brezin, S. Hikami, and J. Zinn-Justin, Generalized nonlinear $\Sigma$ models with gauge invariance, Nucl. Phys. B 165, 528 (1980).

[56] The $\theta$ angle is well defined modulo $2 \pi$ because $\frac{1}{16 \pi} \int \epsilon_{i j} \operatorname{Tr}\left(Q \partial_{i} Q \partial_{j} Q\right)$ is integrally quantized for smooth configurations of $Q$.

[57] A. Pruisken, On localization in the theory of the quantized Hall effect: A two-dimensional realization of the $\theta$-vacuum, Nucl. Phys. B 235, 277 (1984).

[58] H. Levine, S. B. Libby, and A. M. M. Pruisken, Electron Delocalization by a Magnetic Field in Two Dimensions, Phys. Rev. Lett. 51, 1915 (1983).

[59] A. Altland, B. Simons, and M. Zirnbauer, Theories of low-energy quasi-particle states in disordered $d$-wave superconductors, Phys. Rep. 359, 283 (2002).

[60] K. Takahashi and K. B. Efetov, Effect of spin on electron motion in a random magnetic field, Phys. Rev. B 66, 165304 (2002).

[61] P. M. Ostrovsky, I. V. Gornyi, and A. D. Mirlin, Quantum Criticality and Minimal Conductivity in Graphene with Long-Range Disorder, Phys. Rev. Lett. 98, 256801 (2007).

[62] P. M. Ostrovsky, I. V. Gornyi, and A. D. Mirlin, Theory of anomalous quantum Hall effects in graphene, Phys. Rev. B 77, 195430 (2008).

[63] A. Altland, Low-Energy Theory of Disordered Graphene, Phys. Rev. Lett. 97, 236802 (2006).

[64] A. M. Pruisken, Quasi particles in the theory of the integral quantum Hall effect: (II). Renormalization of the Hall conductance or instanton angle theta, Nucl. Phys. B 290, 61 (1987).
[65] Y. B. Kim, A. Furusaki, X.-G. Wen, and P. A. Lee, Gaugeinvariant response functions of fermions coupled to a gauge field, Phys. Rev. B 50, 17917 (1994).

[66] P. Goswami and S. Chakravarty, Superuniversality of topological quantum phase transition and global phase diagram of dirty topological systems in three dimensions, Phys. Rev. B 95, 075131 (2017).

[67] B. L. Altshuler and L. B. Ioffe, Motion of Fast Particles in Strongly Fluctuating Magnetic Fields, Phys. Rev. Lett. 69, 2979 (1992).

[68] D. V. Khveshchenko and S. V. Meshkov, Particle in a random magnetic field on a plane, Phys. Rev. B 47, 12051 (1993).

[69] A. G. Aronov, A. D. Mirlin, and P. Wölfle, Localization of charged quantum particles in a static random magnetic field, Phys. Rev. B 49, 16609 (1994).

[70] An explicit definition of particle-hole symmetry in Dirac composite fermion theory is as follows: $i \rightarrow-i, t \rightarrow-t, \Psi \rightarrow$ $i \sigma^{2} \Psi,\left(a_{0}, a_{j}\right) \rightarrow\left(a_{0},-a_{j}\right),\left(A_{0}, A_{j}\right) \rightarrow\left(-A_{0}, A_{j}\right)$ followed by the shift of the Lagrangian $\mathcal{L}_{D} \rightarrow \mathcal{L}_{D}+\frac{1}{4 \pi} \epsilon^{\mu \nu \rho} A_{\mu} \partial_{v} A_{\rho}$.

[71] C. Nayak, Quantum condensed matter physics-lecture notes.

[72] A. Altland and B. Simons, Condensed Matter Field Theory (Cambridge University Press, Cambridge, 2010).

[73] P. van Nieuwenhuizen and A. Waldron, On euclidean spinors and wick rotations, Phys. Lett. B 389, 29 (1996).

[74] For example, the disorder-averaged retarded Green's function $\overline{G^{R}(\omega, \mathbf{x}, \mathbf{y})}$ equals the $n \rightarrow 0$ limit of the moment $\Psi_{1}(\omega, \mathbf{x}) \Psi_{1}^{\dagger}(\omega, \mathbf{y})$ with respect to $Z$.

[75] P. Streda, Theory of quantised Hall conductivity in two dimensions, J. Phys. C 15, L717 (1982).

[76] J. Goldstone and F. Wilczek, Fractional Quantum Numbers on Solitons, Phys. Rev. Lett. 47, 986 (1981).

[77] X.-L. Qi, T. L. Hughes, and S.-C. Zhang, Topological field theory of time-reversal invariant insulators, Phys. Rev. B 78, 195424 (2008).

[78] K. Fujikawa, Path integral for gauge theories with fermions, Phys. Rev. D 21, 2848 (1980).

[79] I. Affleck, D. Bykov, and K. Wamer, Flag manifold sigma models: spin chains and integrable theories, arXiv:2101.11638.

[80] A. M. M. Pruisken, Dilute instanton gas as the precursor to the integral quantum Hall effect, Phys. Rev. B 32, 2636 (1985).

[81] Y. Huo, R. E. Hetzel, and R. N. Bhatt, Universal Conductance in the Lowest Landau Level, Phys. Rev. Lett. 70, 481 (1993).

[82] R. Gamboa Saravi, M. Muschietti, F. Schaposnik, and J. Solomin, Chiral symmetry and functional integral, Ann. Phys. 157, 360 (1984).

[83] C. Chamon, C.-Y. Hou, R. Jackiw, C. Mudry, S.-Y. Pi, and G. Semenoff, Electron fractionalization for two-dimensional dirac fermions, Phys. Rev. B 77, 235431 (2008). 